This is the final peer-reviewed accepted manuscript of:

Chesani, Federico et al. 'Compliance in Business Processes with Incomplete Information and Time Constraints: a General Framework Based on Abductive Reasoning'. 1 Jan. 2018 : $75-111$.

The final published version is available online at: http://dx.doi.org/10.3233/FI-20181696

Rights / License:

The terms and conditions for the reuse of this version of the manuscript are specified in the publishing policy. For all terms of use and more information see the publisher's website. 


\title{
Compliance in Business Processes with Incomplete Information and Time Constraints: a General Framework based on Abductive Reasoning*
}

\author{
Federico Chesani, Paola Mello \\ University of Bologna \\ viale Risorgimento 2, 40136-Bologna, Italy \\ $\{$ federico.chesani_paola.mello\}@unibo.it
}

\author{
Marco Montali*, Sergio Tessaris \\ Free University of Bozen-Bolzano \\ piazza Università, 1, 39100 Bozen-Bolzano, Italy \\ \{montali_tessaris\}@inf.unibz.it
}

\author{
Riccardo De Masellis ${ }^{\dagger}$, Chiara Di \\ Francescomarino*, Chiara Ghidini* \\ FBK-IRST \\ Via Sommarive 18, 38050 Trento, Italy \\ $\{$ r.demasellis — dfmchiara_ghidini $\} @ f b k . e u$
}

\begin{abstract}
The capability to store data about Business Process (BP) executions in so-called Event Logs has brought to the identification of a range of key reasoning services (consistency, compliance, runtime monitoring, prediction) for the analysis of process executions and process models. Tools for the provision of these services typically focus on one form of reasoning alone. Moreover, they are often very rigid in dealing with forms of incomplete information about the process execution. While this enables the development of ad hoc solutions, it also poses an obstacle for the adoption of reasoning-based solutions in the BP community.

In this paper, we introduce the notion of Structured Processes with Observability and Time (SPOT models), able to support incompleteness (of traces and logs), and temporal constraints on the activity duration and between activities. Then, we exploit the power of abduction to provide a flexible, yet computationally effective framework able to reinterpret key reasoning services in terms of incompleteness and observability in a uniform way.
\end{abstract}

Keywords: Business Processes, Incomplete traces, Observability, Temporal workflows, Abductive Logic Programming

Address for correspondence: Federico Chesani, University of Bologna, viale Risorgimento 2, 40136-Bologna, Italy. E-Mail: federico.chesani@unibo.it

*This work is an extended version of a preliminary, position paper presented at the ECAI2016 conference [1].

${ }^{\dagger}$ This research has partially been carried out within the Euregio IPN12 KAOS, which is funded by the "European Region Tyrol-South Tyrol-Trentino" (EGTC) under the first call for basic research projects. 


\section{Introduction}

The proliferation of IT systems able to store process executions traces in so-called event logs has originated, in the Business Process (BP) community, a quest towards tools that offer the possibility of discovering, checking the conformance and enhancing process models based on actual behaviors [2]. Focusing on conformance, that is, on a scenario where the aim is to assess how a prescriptive (or "de jure") process model relates to the execution traces, this general notion can be declined in specific "use cases", such as model consistency, trace compliance, runtime monitoring and prediction/recommendation [3]. These reasoning services are often investigated in isolation and tailored to specific workflow languages. Thus [4] assesses runtime monitoring over processes with business constraints, while [5] assesses model consistency with business contracts.

While the construction of tools that offer specific reasoning services over specific workflow languages enables the development of ad hoc effective solutions, it also poses an obstacle for the adoption of reasoning-based solutions in the BP community. In fact, these tools often cover only few of the "use cases" and do not easily adapt to different workflow languages or to event logs containing execution traces with different characteristics. This poses a problem, given the current trends of (i) enriching business process modelling languages with new constructs complementing the control flow knowledge, and (ii) extending the notion of conformance to more and more realistic event logs.

Two important examples of extension of the modelling language and of extension of the event $\log$ nature, relevant for the BPM community, are temporal workflows and partial event logs. Temporal workflows, i.e., workflows enriched with constructs for temporal constraints, are investigated in a stream of recent work. [6, 7, 8], for instance, address modelling and conformance-related aspects of business process models enriched with temporal constraints, providing "ex-novo" investigations of reasoning services such as model consistency and runtime monitoring/prediction. An analogous stream of recent papers is devoted to the problem of dealing with partial event logs, where the execution traces may bring incomplete information about the process execution. Examples are [9, 10, 11]. All these works address the problem of "repairing" (i.e., completing) incomplete execution traces, exploiting different "ad-hoc" techniques based on different approaches such as replay-based techniques, automated planning, and the integration of trace information with data coming from further sources, respectively.

Artificial Intelligence has a long tradition providing frameworks able to integrate diverse reasoning tasks in the presence of incomplete information. A paradigmatic setting is Abductive Logic Programming (ALP) [12], where the integration of constraint solving features (ACLP) [13] has enhanced its practical utility by allowing expressive and flexible representations of the problem domain, and by making the abductive computation more efficient and powerful. Abduction fits in a natural manner with the scenario of execution traces: facts are observed in the execution traces, and need to be explained/diagnosed with respect to what is envisaged by the process model. This is indeed strictly related with the definition of abductive reasoning.

In this work we exploit the paradigm of ACLP [13], and the SCIFF abductive framework [14] to provide a general purpose environment able to support conformance in its different "use cases" in the presence of two important variants of workflow models and event logs, namely temporal constraints on activity durations and between different activities, and incomplete event data. The novel contribution 


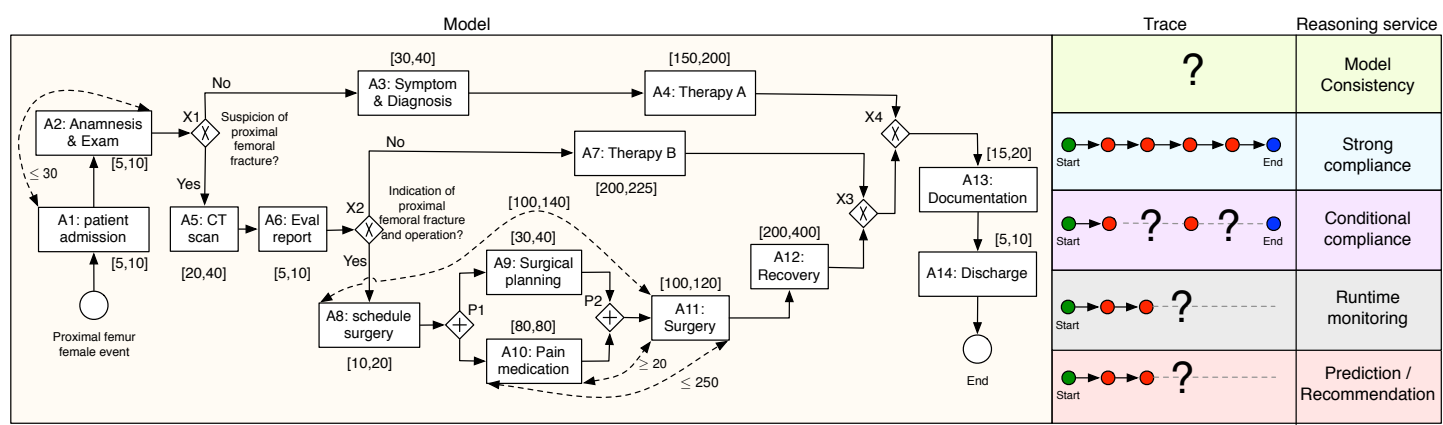

Figure 1. A process for femoral fracture treatment taken from [7], and reasoning services and incomplete execution traces.

of the paper is as follows. First, we provide a formal definition of the scenario at hand, by introducing the notion of Structured Processes with Observablity and Time (SPOT models), and by reformulating business process reasoning services in terms of incompleteness (Sec. 2). This produces a refinement of the classical notion of compliance into strong, and conditional compliance to take into account different sources of incomplete knowledge in the analysed traces. Second, an encoding of SPOT models and event logs in SCIFF is provided. To show the flexibility of our approach to capture different workflow languages in a modular manner, we address structured process models enriched with temporal aspects (Sec. 3.1). The SCIFF proof procedure is then exploited and evaluated in Sec. 3.2, 3.3, and 4 .

\section{Process Models, Reasoning Services and Incompleteness}

Given the prescriptive knowledge contained in a well-structured process mode $\rfloor^{1}$ enriched with temporal constraints, we aim at understanding how to re-interpret typical reasoning services as reasoning on incomplete traces.

\subsection{Process Models and Incomplete Traces}

We illustrate our investigation with an example of temporal workflow taken from [7], and reproduced in the left hand side of Figure $\left.1\right|^{2}$ This workflow contains: 14 activities $(A 1, \ldots A 14), 2$ pairs of exclusive gateways $(\langle X 1, X 4\rangle,\langle X 2, X 3\rangle)$, and 1 pair of parallel gateways $(\langle P 1, P 2\rangle)$. In addition, the language contains constructs to denote temporal information: activities are labelled with expressions of the form $\left[d_{\min }, d_{\max }\right]$ indicating the duration range of the activity ${ }^{3}$, and dashed arrows between

\footnotetext{
${ }^{1}$ We focus on structured process models in the spirit of [15]. Broadly speaking, this restricts to the class of models recursively composed of single-entry-single-exit blocks, where every split has a corresponding join, matching its type. This assumption rules out pathological patterns that are notoriously hard to characterise (e.g. involving nested OR joins), still providing coverage for a wide range of interesting use cases.

${ }^{2}$ Workflow models are rendered using the widely adopted BPMN graphical notation (see www.bpmn.org).

${ }^{3}$ In the remainder of this paper we will assume that the time domain relies on natural numbers. Alternative temporal domains can be seamlessly studied.
} 
activities expresses inter-task constraints involving the start or end of the activities; depending on the position of the arrow w.r.t. the box representing the activity. As an example, the dashed arrow between $A 1$ and $A 2$ indicates that a constraint of at most 30 time units exists between the start of $A 1$ and the end of $\mathrm{A} 2$.

We assume that each execution of this process, hereafter called the Femur-Fracture (FF) process, is logged by an information system. We also assume that activities have a time span e.g., event $\left(\mathrm{A},\left[T_{s}, T_{e}\right]\right)$ indicates that activity $\mathrm{A}$ has been executed from $T_{s}$ to $T_{e}$. A sample trace that $\operatorname{logs}$ the execution of a FF instance is:

$$
\{(\mathrm{A} 1,[2,7]),(\mathrm{A} 2,[10,15]),(\mathrm{A} 3,[16,46]),(\mathrm{A} 4,[50,200]),(\mathrm{A} 13,[300,317]),(\mathrm{A} 14,[320,330])\}
$$

A multiset of traces of the same process forms an event log.

In the aforementioned trace, all information have been explicitly logged. To incorporate incompleteness into the picture, the process models we consider are also equipped with observability information. This information provides a fine-grained characterization of different levels of incompleteness in the activities present in the process model, when it comes to "logging" the execution of such activities when running the process. In particular, three observability categories are considered:

- An observable activity is an activity that is explicitly logged. Here incompleteness may arise because some information (i.e., the activity name, the starting time, the ending time, or a combination thereof) may be missing.

- A non-observable activity is an activity that is never logged. Here incompleteness arises because such activity could have been actually executed, even though this is not traced in the log.

- A partially observable activity is an activity that may or may not be logged, depending on the context. In other words, some traces may contain an explicitly logged entry witnessing the execution of such an activity, while in others this could be missing.

Formally, we extend the approach in [7] with observability information, obtaining the following model. First we introduce the notion of Process block to describe structured processes.

\section{Definition 2.1. (Process Block)}

A process block is inductively defined as follows:

(base case) a $\in \mathcal{A}$ is a process block, called task block;

(inductive case) $\left\langle\right.$ type, $\left.B_{1}, B_{2}\right\rangle$ is a process block, where type $\in\{$ seq, xor, and, or $\}$ indicates the

block type (where seq stands for sequence, xor for exclusive choice, and for parallel split, and or for inclusive split), while $B_{1}$ and $B_{2}$ are process blocks.

The definition of blocks may be directly extended to the case of $n$ sub-blocks instead of just 2 . In particular, in the following we make use of a ternary sequence block $\left\langle\right.$ seq, $\left.B_{1}, B_{2}, B_{3}\right\rangle$.

We say that block $B_{1}$ is in block $B_{2}$ if $B_{1}$ belongs to a sub-tree of such tree that is rooted in $B_{1}$.

Furthermore, it is well-known that an inclusive split block can be reformulated in terms of a choice considering the execution of the first inner-block only, the execution of the second inner-block only, or the parallel execution of both inner-blocks. Hence, from now on we will omit the case of inclusive blocks.

\section{Definition 2.2. (Structured Process with Observability and Time)}

A structured process with observability and time (SPOT) is a tuple $\langle\mathcal{A}$, obs, $P$, dur, tcon $\rangle$, where: 
- $\mathcal{A}$ is a finite set of activity (names);

- obs : $\mathcal{A} \rightarrow\{\mathrm{o}, \mathrm{n}, \mathrm{p}\}$ is a total observability function, indicating for each activity in $\mathcal{A}$ whether it is observable (o), non-observable (n), or partially observable (p).

- $P$ is the top process block

- dur : $\mathcal{A} \rightarrow \mathbb{N}^{+} \times \mathbb{N}^{+} \cup\{\infty\}$ is a total duration function, assigning a duration interval to each activity in $\mathcal{A}$, where $\operatorname{dur}(\mathrm{a})=\langle m, n\rangle$ means that the duration of a is between $m$ and $n$ time units. We assume that for each activity $\mathrm{t} \in \mathcal{A}$ with $\operatorname{dur}(\mathrm{t})=\langle m, n\rangle, n$ is either $\infty$ or a number $\geq m$. We use $\infty$ as a special symbol to indicate that the maximum duration is unconstrained 4

- tcon $: \mathcal{A} \times\{\mathfrak{s}, \mathfrak{e}\} \times \mathcal{A} \times\{\mathfrak{s}, \mathfrak{e}\} \rightarrow \mathbb{N}^{+} \times \mathbb{N}^{+} \cup\{\infty\}$ is a partial inter-task constraint function, indicating the expected duration interval that can intervene between the start/end of an activity and the start/end of another activity, where the start is marked with symbol $\mathfrak{s}$, and the end is marked with symbol e. E.g., $\operatorname{tcon}\left(\mathrm{a}_{1}, \mathfrak{e}, \mathrm{a}_{2}, \mathfrak{s}\right)=\langle m, n\rangle$ if the time duration intervening between the end of $\mathrm{a}_{1}$ and the start of $\mathrm{a}_{2}$ belongs to the time interval $[m, n]$.

We assume that $P$ is well-defined, in the sense that each activity a $\in \mathcal{A}$ appears in exactly one block. This is a standard assumption guaranteeing that no process block can directly or indirectly refer to itself, and also that each task is unambiguously located within the process. It also implies that the sub-block relation induces a tree rooted in $P$ and whose leaves are activities from $\mathcal{A}$.

Example 2.3. Consider the fragment of temporal workflow constituted by the first two tasks in Figure 11, so that the first task is observable, whereas the second is not. It corresponds to SPOT $\langle\mathcal{A}, o b s, P, d u r, t c o n\rangle$, where: $(i) \mathcal{A}=\{\mathrm{A} 1, \mathrm{~A} 2\}$, (ii) obs is such that $o b s(\mathrm{~A} 1)=\mathrm{o}$ and $o b s(\mathrm{~A} 2)=\mathrm{n}$, (iii) $P=\langle$ seq, A1, A2 $\rangle$, (iv) dur is such that $\operatorname{dur}(\mathrm{A} 1)=\operatorname{dur}(\mathrm{A} 2)=\langle 5,10\rangle$, (v) tcon is such that $\operatorname{tcon}(\mathrm{A} 1, \mathfrak{s}, \mathrm{A} 2, \mathfrak{e})=\langle 0,30\rangle$.

The omission of loop blocks in Definition 2.1 is due to the the unclear semantics of the interplay between loops and inter-task constraints. In fact, loops are excluded from temporal workflows introduced in the literature (e.g. [7, 16]). In Section 5] we discuss in more details these semantic ambiguities and we show how loops can be incorporated in our framework.

From now on, we assume that the SPOT of interest is structured in the following normal form.

\section{Definition 2.4. (Normal SPOT)}

A SPOT $\langle\mathcal{A}$, obs, $P$, dur, tcon $\rangle$ with $\mathcal{A}=\mathcal{A}_{a} \uplus \mathcal{A}_{b}$ is in normal form (hence, a normal SPOT) if:

- Task blocks in $P$ use only activities from $\mathcal{A}_{a}$.

- Set $\mathcal{A}_{b}$ is the smallest set satisfying the following condition: for each block $B$ in $P, \mathcal{A}_{b}$ contains two special, atomic activities in ${ }_{B}$ and out . .

- For every activity $\mathrm{b} \in \mathcal{A}_{b}$, we have that $\operatorname{obs}(\mathrm{b})=\mathrm{n}$, and $\operatorname{dur}(\mathrm{b})=\langle 0,0\rangle$.

- $P$ has the form $\left\langle\right.$ seq, in $P, P^{\prime}$, out $\left.P\right\rangle$, and every inductive block of the form $\left\langle\right.$ type, $\left.B_{1}, B_{2}\right\rangle$ in $P^{\prime}$ is such that $B_{i}$ has the form $\left\langle\right.$ seq, in $_{B_{i}}, B_{i}^{\prime}$, out $\left.B_{i}\right\rangle$ for $i \in\{1,2\}$.

- Function tcon only mentions activities in $\mathcal{A}_{a}$.

Intuitively, in a normal SPOT each block is associated to two special activities that mark the entry and exit points into/outside the block. Such activities are atomic and nonobservable, and are used to

\footnotetext{
${ }^{4}$ Thus, if activity a has unconstrained duration, we have $\operatorname{dur}(\mathrm{a})=\langle 0, \infty\rangle$.
} 
"interleave" the nesting of blocks. An arbitrary SPOT $\mathcal{P}$ can be straightforwardly converted in normal form, by introducing dedicated special activities capturing entrance/exit into/from its blocks, and then recursively "normalizing" its top block by replacing each block with its normalized version.

We next define the notion of trace, taking into account the presence of missing information units, denoted with the special symbol ".".

\section{Definition 2.5. ((SPOT) trace)}

A (SPOT) trace over a set $\mathcal{A}$ of activities is a finite set of event triples $\langle\mathrm{a}, s, e\rangle$, where a $\in \mathcal{A} \uplus\left\{{ }_{-}\right\}$ is an activity, and $s, e \in \mathbb{N} \uplus\{-\}$ are the start and end timestamps of the event. A trace is partially specified if some of its event triples contain the special symbol "., fully specified otherwise.

As in our running example, we represent event triples using notation $(\mathrm{a},[s, e])$. When a is atomic, i.e., $s=e$, we simply write $(\mathrm{a}, s)$. We say that activity a occurs in trace $\mathcal{T}$ if there exist $s, e \in \mathbb{N} \uplus\left\{{ }_{-}\right\}$ such that $(\mathrm{a},[s, e]) \in \mathcal{T}$. Notice that, independently of whether a trace is fully or partially specified, there is another dimension of incompleteness, which accounts for the distinction between real and logged traces. In a logged trace, additional event triples may be implicitly present even if not explicitly listed in the trace. This is the case when the process model of interest contains activities that are not always observable. In this light, a real trace fully details a process execution, while a partial trace its observable counterpart. Furthermore, different real traces correspond to different completions of the same logged trace.

\section{Definition 2.6. (Logged trace)}

A logged trace of $\operatorname{SPOT}\langle\mathcal{A}, o b s, P, d u r, t c o n\rangle$ is a trace over $\{\mathrm{a} \mid \mathrm{a} \in \mathcal{A}$ and $o b s(\mathrm{a}) \in\{\mathrm{o}, \mathrm{p}\}\}$.

\section{Definition 2.7. (Possible completion)}

Let $\mathcal{P}=\langle\mathcal{A}$, obs, $P$, dur, tcon $\rangle$ be a SPOT, and $\mathcal{T}$ be a logged trace of $\mathcal{P}$. Trace $\overline{\mathcal{T}}$ is a possible completion of $\mathcal{T}$ for $\mathcal{P}$ over tasks $\mathcal{A}_{c} \subseteq \mathcal{A}$ if $\overline{\mathcal{T}}$ is a fully specified trace over $\mathcal{A}_{c}$, and $\overline{\mathcal{T}}=\mathcal{T}_{\text {attr }} \uplus \mathcal{T}_{\text {ext }}$, where:

1. Trace $\mathcal{T}_{\text {attr }}$, called possible attribute completion of $\mathcal{T}$ for $\mathcal{P}$ over tasks $\mathcal{A}_{c}$, is the smallest fully specified trace satisfying the following condition: for every event $\left(X,\left[T_{1}, T_{2}\right]\right) \in \mathcal{T}$, there exist an activity a $\in \mathcal{A}_{c}$ and two timestamps $s, e \in \mathbb{N}$ such that: (i) $(\mathrm{a},[s, e]) \in \overline{\mathcal{T}}$, (ii) if $X \in \mathcal{A}$, then $X=\mathrm{a}$, (iii) if $T_{1} \in \mathbb{N}$, then $T_{1}=s$, (iv) if $T_{2} \in \mathbb{N}$, then $T_{2}=e 5^{5}$

2. Trace $\mathcal{T}_{\text {ext }}$, called possible extension of $\mathcal{T}$ for $\mathcal{P}$ over tasks $\mathcal{A}_{c}$, contains additional events that only refer to unobservable or partially-observable activities from $\mathcal{A}_{c}$.

Intuitively, the separation between attribute completion and extension is useful to isolate the portion of a completion that mirrors the original trace, grounding its unspecified elements, from the portion that genuinely extends it with further events. Notice that in every completion of a fully specified trace, the attribute completion always coincides with the trace itself.

The fact that a completion may be defined over just a subset of all the available activities in a SPOT is useful to distinguish between unobservable domain tasks and unobservable artificial tasks (such as those introduced in normal SPOTs to mark the boundaries of blocks).

\footnotetext{
${ }^{5}$ Recall that a and/or $s$ and/or $e$ may be filled with “.”.
} 


\subsection{Compliance}

Several fundamental reasoning services can be studied over SPOTs (cf. the right-hand side of Figure 11. We start by defining a basic notion of trace compliance, which mirrors the intended execution semantics of SPOTs. Recall that we are employing the normal form for SPOTs.

\section{Definition 2.8. (Block compliance)}

Let $\mathcal{A}$ be a set of activities. A fully specified trace $\mathcal{T}$ over $\mathcal{A}$ complies with a block $B=$ $\left\langle\right.$ seq, $\left\langle\operatorname{in}_{B}, B^{\prime}\right.$, out $\left.\left._{B}\right\rangle\right\rangle$ if either both activities $\operatorname{in}_{B}$ and out $_{B}$ occur in $\mathcal{T}$ or none does, and one of the following conditions hold:

1. (i) $B^{\prime}=$ a with a $\in \mathcal{A}$, (ii) in in $_{B}$ occurs in $\mathcal{T}$ if and only if a occurs in $\mathcal{T}$, (iii) if there exist $t_{i}, t_{s}, t_{e}, t_{o} \in \mathbb{N}$ such that $\left\{\left(\right.\right.$ in $\left._{B}, t_{i}\right),\left(\mathrm{a},\left[t_{s}, t_{e}\right]\right),\left(\right.$ out $\left.\left._{B}, t_{o}\right)\right\} \subseteq \mathcal{T}$, then $t_{i} \leq t_{s}<t_{e}=t_{o}$.

2. (i) $B^{\prime}=\left\langle\right.$ seq, $\left.B_{1}, B_{2}\right\rangle$, (ii) $B_{1}$ and $B_{2}$ are compliant with $\mathcal{T}$, (iii) in in $_{B}$ occurs in $\mathcal{T}$ if and only if in $_{B_{1}}$ occurs in $\mathcal{T}$ if and only if $\operatorname{in}_{B_{2}}$ occurs in $\mathcal{T}$, (iv) if there exist $t_{i}, t_{i 1}, t_{o 1}, t_{i 2}, t_{o 2}, t_{o} \in$ $\mathbb{N}$ such that $\left\{\left(\right.\right.$ in $\left._{B}, t_{i}\right),\left(\right.$ in $\left._{B_{1}}, t_{i 1}\right),\left(\right.$ out $\left._{B_{1}}, t_{o 1}\right),\left(\right.$ in $\left._{B_{2}}, t_{i 2}\right),\left(\right.$ out $\left._{B_{2}}, t_{o 2}\right),\left(\right.$ out $\left.\left._{B}, t_{o}\right)\right\} \subseteq \mathcal{T}$, then $t_{i}=t_{i 1} \leq t_{o 1}=t_{i 2} \leq t_{o 2}=t_{o}$.

3. (i) $B^{\prime}=\left\langle\right.$ and, $\left.B_{1}, B_{2}\right\rangle$, (ii) $B_{1}$ and $B_{2}$ are compliant with $\mathcal{T}$, (iii) in in $_{B}$ occurs in $\mathcal{T}$ if and only if in $_{B_{1}}$ occurs in $\mathcal{T}$ if and only if in $B_{2}$ occurs in $\mathcal{T}$, (iv) if there exist $t_{i}, t_{i 1}, t_{o 1}, t_{i 2}, t_{o 2}, t_{o} \in$ $\mathbb{N}$ such that $\left\{\left(\right.\right.$ in $\left._{B}, t_{i}\right),\left(\right.$ in $\left._{B_{1}}, t_{i 1}\right),\left(\right.$ out $\left._{B_{1}}, t_{o 1}\right),\left(\right.$ in $\left._{B_{2}}, t_{i 2}\right),\left(\right.$ out $\left._{B_{2}}, t_{o 2}\right),\left(\right.$ out $\left.\left._{B}, t_{o}\right)\right\} \subseteq \mathcal{T}$, then $t_{i}=t_{i 1}=t_{i 2} \leq t_{o 1}, t_{i} \leq t_{o 2}$, and $t_{o}=\max \left(t_{o 1}, t_{o 2}\right)$.

4. (i) $B^{\prime}=\left\langle\right.$ xor, $\left.B_{1}, B_{2}\right\rangle$, (ii) $B_{1}$ and $B_{2}$ are compliant with $\mathcal{T}$, (iii) in in $_{B}$ occurs in $\mathcal{T}$ if and only if either $\operatorname{in}_{B_{1}}$ or $\operatorname{in}_{B_{2}}$ occur in $\mathcal{T}$, (iv) it is not the case that $\operatorname{in}_{B_{1}}$ and in $_{B_{2}}$ both occur in $\mathcal{T}$, (v) if there exist $t_{i}, t_{i j}, t_{o j}, t_{o} \in \mathbb{N}$ with $j \in\{1,2\}$, such that $\left\{\left(\right.\right.$ in $\left._{B}, t_{i}\right),\left(\right.$ in $\left._{B j}, t_{i j}\right),\left(\right.$ out $\left._{B j}, t_{j}\right),\left(\right.$ out $\left.\left._{B}, t_{o 2}\right)\right\} \subseteq \mathcal{T}$, then we have $t_{i}=t_{i j} \leq t_{o j}=t_{o}$.

Intuitively, Definition 2.8 states that a trace complies with a block if it does not go through the block at all, or if the trace enters the the block, then it also exits from the block, and suitably traverses it. Suitably traversing, in turn, depends on the block type. If the block is a task block, then the inner activity has to be executed after the trace enters into the block, and the block is completed as soon as the activity is finished. If the block is a sequence block, then the trace has to go through the inner blocks sequentially, and must comply with both. If the block is a parallel block, then the trace has to go through the inner blocks in whatever sequence, and must comply with both; in addition, the trace must exit from the block at the exact time when the latest of the two inner blocks is completed. Finally, if the block is a choice block, the execution has to go through exactly one of the inner blocks, avoiding the other one; in addition, the trace has to comply with the inner blocks (this is vacuously true for the block that is not chosen, since such block is not even entered).

\section{Definition 2.9. (Duration compliance)}

Let $\mathcal{A}$ be a set of activities, and $d u r$ a duration function over $\mathcal{A}$. A fully specified trace $\mathcal{T}$ over $\mathcal{A}$ complies with dur if, for each activity a $\in \mathcal{A}$ with $\operatorname{dur}(\mathrm{a})=\langle m, n\rangle$, whenever there exist $s, e \in \mathbb{N}$ s.t. $($ a, $[s, e]) \in \mathcal{T}$, we have $m \leq e-s \leq n$.

\section{Definition 2.10. (Inter-task constraint compliance)}

Let $\mathcal{A}$ be a set of activities, and tcon an inter-task constraint function. A fully specified trace $\mathcal{T}$ 
over $\mathcal{A}$ complies with tcon if, for every pair of activities $\mathrm{a}, \mathrm{b} \in \mathcal{A}$ and $s_{a}, e_{a}, s_{b}, e_{b} \in \mathbb{N}$ such that $\left\{\left(\mathrm{a},\left[s_{a}, e_{a}\right]\right),\left(\mathrm{b},\left[s_{b}, e_{b}\right]\right)\right\} \in \mathcal{T}$, the following conditions hold:

- if $\operatorname{tcon}(\mathrm{a}, \mathfrak{s}, \mathrm{b}, \mathfrak{s})=\left\langle m_{1}, n_{1}\right\rangle$, then $m_{1} \leq s_{b}-s_{a} \leq n_{1}$;

- if $\operatorname{tcon}(\mathrm{a}, \mathfrak{s}, \mathrm{b}, \mathfrak{e})=\left\langle m_{2}, n_{2}\right\rangle$, then $m_{2} \leq e_{b}-s_{a} \leq n_{2}$;

- if $\operatorname{tcon}(\mathrm{a}, \mathfrak{e}, \mathrm{b}, \mathfrak{s})=\left\langle m_{3}, n_{3}\right\rangle$, then $m_{3} \leq s_{b}-e_{a} \leq n_{3}$;

- if $\operatorname{tcon}(\mathrm{a}, \mathfrak{e}, \mathrm{b}, \mathfrak{e})=\left\langle m_{4}, n_{4}\right\rangle$, then $m_{4} \leq e_{b}-e_{a} \leq n_{4}$.

When a trace does not comply with a block or duration/inter-task constraint, we say that the trace violates such a block/constraint. Definitions 2.9 and 2.10 impose that the trace respects the modeled temporal constraints, respectively ensuring that the duration of each activity execution agrees with the specified duration, and that whenever the trace contains two activities that are subject to an inter-task constraint, the time distance between their execution must agree with the specified constraint.

We put together the three definitions above, defining a general notion of strong compliance.

\section{Definition 2.11. (Strong compliance)}

Let $\mathcal{P}=\langle\mathcal{A}$, obs, $P$, dur, tcon $\rangle$ be a normal SPOT, with $\mathcal{A}=\mathcal{A}_{a} \uplus \mathcal{A}_{b}$. A logged trace $\mathcal{T}$ over $\mathcal{A}_{a}$ is strongly compliant with $\mathcal{P}$ if: (i) $\mathcal{T}$ is fully specified; (ii) $\mathcal{T}$ is compliant with dur and tcon; (iii) there exists a completion $\overline{\mathcal{T}}$ of $\mathcal{T}$ for $\mathcal{P}$ over $\mathcal{A}_{b}$ such that in ${ }_{P}$ occurs in $\overline{\mathcal{T}}$, and $\overline{\mathcal{T}}$ is compliant with $P$. In this case, we say that $\overline{\mathcal{T}}$ is a strong compliance witness for $\mathcal{T}$ w.r.t. $\mathcal{P}$.

We stress two aspects of this definition. First, since the logged trace $\mathcal{T}$ is fully specified, every completion $\overline{\mathcal{T}}$ can only extend $\mathcal{T}$ with further events. Second, since $\overline{\mathcal{T}}$ extends $\mathcal{T}$ only over $\mathcal{A}_{b}$, such an extension can only consists of artificial events referring to entering into/exiting from blocks.

This notion of compliance is used to characterise the degree to which a given trace conforms/is aligned to the model. In fact, it reflects the usual notion of compliance for business processes, where compliance is typically defined under the assumption that the trace represents a complete end-to-end execution that can be fully replayed on the process model. This is why we call it "strong" compliance. More specifically, the first condition in Definition 2.11 imposes that the trace of interest only contains executions that may be observed. Hence, processes that prescribe the unavoidable execution of unobservable tasks do not admit logged traces that are strongly compliant. The second condition imposes that the execution times of the activity executions present in the trace respect the durations and inter-task constraints of the model. The last condition indicates that the trace complies with the considered process. This amounts to block compliance, once the input trace is suitably augmented with additional events that refer to the (unobservable) special activities used to mark the boundaries of blocks. Among those events, we also require to enter the top block, i.e., to start the overall process.x

An example of strong compliant trace is the one in (1). Replacing $(A 2,[10,15])$ with $(A 2,[50,60])$ in (1) makes it become not compliant. In fact, going from the start of A1 to the end of A2 would require 58 time units thus violating the inter-task constraint between these two activities.

\subsection{The Additional Reasoning Services}

Starting from the notion of (strong) compliance, we define the remaining reasoning tasks of Figure 1 (right hand side), covering the classical notion of consistency, as well as different notions of compliance that take into account the sources of incompleteness that may be present in the trace. 
Conditional compliance handles the case where the trace under analysis is indeed partial and/or partially specified. This source of incompleteness in a trace hinders the possibility of replaying it on the process model. However, strong compliance might be regained once the trace is augmented additional information on missing or partially specified events that refer to tasks that are or may be unobserved.

\section{Definition 2.12. (Conditional compliance)}

Let $\mathcal{P}=\langle\mathcal{A}$, obs, $P$, dur, tcon $\rangle$ be a normal SPOT, with $\mathcal{A}=\mathcal{A}_{a} \uplus \mathcal{A}_{b}$. A logged trace $\mathcal{T}$ over $\mathcal{A}_{a}$ is conditionally compliant with $\mathcal{P}$ if there exists a completion $\overline{\mathcal{T}}$ of $\mathcal{T}$ for $\mathcal{P}$ over $\mathcal{A}_{a}$ that is strongly compliant with $\mathcal{P}$. In this case, we say that $\overline{\mathcal{T}}$ is a conditional compliance witness for $\mathcal{T}$ w.r.t. $\mathcal{P}$.

It is worth noting that, in the definition of conditional compliance, the completion of the given trace is defined only over the genuine tasks of the input SPOT, not the artificial ones marking the boundaries of process blocks. If the given logged trace is fully specified, then the only completion of this form is the trace itself, and consequently conditional compliance coincides with strong compliance. If instead the given logged trace is partially specified, then multiple (even infinitely many) completions witnessing conditional compliance may exist.

A sample partial trace over the FF model is:

$$
\left\{(\mathrm{A} 1,[2,7]),\left(\mathrm{A} 2,_{-}\right),(\mathrm{A} 4,[50,-]),(\mathrm{A} 13,[300,317]),(\mathrm{A} 14,[320,330])\right\}
$$

Notice that " " may refer to a missing event name (_, $[1,3])$, missing event time $\left(A 2,_{-}\right)$or missing event time detail $(\mathrm{A} 4,[50,-])$. It is easy to see that 2 is compliant with $\mathrm{FF}$, if

A2 was executed in an interval $\left[T 2_{s}, T 2_{e}\right]$ s.t. $7<T 2_{s}$;

$$
T 2_{e}<50 ; 5 \leq T 2_{e}-T 2_{s} \leq 10 \text { and } T 2_{e}-2 \leq 30
$$

an execution of $\mathrm{A} 3$ was performed in an interval $\left[T 3_{s}, T 3_{e}\right]$

s.t. $T 2_{e}<T 3_{s} ; T 3_{e}<50 ;$ and $30 \leq T 3_{e}-T 3_{s} \leq 40$

A4 was executed with end time $T 4_{e}$ s.t. $T 4_{e}<300$

$$
\text { and } 150 \leq T 4_{e}-50 \leq 200
$$

Summing up, a trace $\widehat{\mathcal{T}}$ as defined in Definition 2.12, that would make the trace $\mathcal{T}$ in 2 conditionally complaint would be:

$$
\{(\mathrm{A} 1,[2,7]),(\mathrm{A} 2,[10,17]),(\mathrm{A} 3,[18,48]),(\mathrm{A} 4,[50,220]),(\mathrm{A} 13,[300,317]),(\mathrm{A} 14,[320,330])\}
$$

Note that the set of assumptions needed to reconstruct full conformance is not necessarily unique. This because alternative strongly compliant real process executions might have led to the recorded partial trace. On the other hand, there are situations in which it is impossible to recover compliance formulating additional assumptions. In this case, the partial trace is considered non-compliant. E.g.,

$$
\left\{\left(\mathrm{A} 1,\left[T 1_{s}, T 1_{e}\right]\right),\left(\mathrm{A} 3,_{-}\right)\left(\mathrm{A} 9,\left[-, T 9_{e}\right]\right)\right\}
$$

does not comply with FF since A3 and A9 belong to mutually exclusive branches in the model.

With strong and conditional compliance at hand, we can define the general notion of compliance. 
Definition 2.13. Let $\mathcal{P}=\langle\mathcal{A}$, obs, $P$, dur, tcon $\rangle$ be a normal SPOT, with $\mathcal{A}=\mathcal{A}_{a} \uplus \mathcal{A}_{b}$. A logged trace $\mathcal{T}$ over $\mathcal{A}_{a}$ is compliant with $\mathcal{P}$ if there exists a completion $\overline{\mathcal{T}}$ of $\mathcal{T}$ for $\mathcal{P}$ over the entire set $\mathcal{A}$ of activities that satisfies the following conditions: $(i) \overline{\mathcal{T}}$ is compliant with $d u r$ and tcon; $(i i)$ in $_{P}$ occurs in $\overline{\mathcal{T}}$; (iii) $\overline{\mathcal{T}}$ is compliant with $P$. In this case, we say that $\overline{\mathcal{T}}$ is a compliance witness for $\mathcal{T}$ w.r.t. $\mathcal{P}$.

Model Consistency checks if a SPOT enables acceptable executions from start to end. This case bears no (that is, fully incomplete) information on the execution traces, and reasons on the model alone to determine whether it has acceptable executions.

\section{Definition 2.14. (Consistency)}

A SPOT $\mathcal{P}$ is consistent if the empty trace is conditionally compliant with $\mathcal{P}$.

Figure 1 shows a consistent model. Modifying the inter-task constraint between the start of A1 to the end of $A 2$ replacing $\leq 30$ with $\leq 5$ makes the model inconsistent. In fact executing $A 1$ and $A 2$ in sequence requires at least 10 units of time.

While compliance refers to terminated traces, runtime monitoring aims at dealing with ongoing executions in order to detect early violations of compliance / ensure the existence of a positive outcome. Here the incompleteness concerns future steps of an ongoing process execution as in the following trace whose last activity executed is $A 9$ :

$$
\{(\mathrm{A} 1,[2,7]),(\mathrm{A} 2,[10,15]),(\mathrm{A} 5,[16,46]),(\mathrm{A} 6,[50,60]),(\mathrm{A} 8,[200,210]),(\mathrm{A} 9,[350,380])\}
$$

This trace already violates the inter-task constraint between $A 8$ and $A 11$ (even if $A 11$ has not been executed yet). In fact 170 units of time have already passed between the start of T8 and the end of T9 while an inter-task constraint requires that A11 starts at most 140 units of time after the start of A8. In the case of an evolving trace, conditional compliance can be directly defined by extending Def. 2.12 so that also events referring to observable activities in $\widehat{\mathcal{T}}$ can be added providing that these are hypothesized to occur at times that go beyond the current time (i.e., no observable event can be hypothesized in the past).

Similarly to runtime monitoring, prediction/recommendation deals with ongoing executions with the aim of providing a completion that satisfies certain conditions. For example, consider a process execution composed of the first 5 events of (8) (that is, surgery has been scheduled). One could ask a recommender system to provide the sequence of actions that minimize the time needed to discharge the patient and terminate the process, obtaining the following answer:

$$
\begin{aligned}
& \{(\mathrm{A} 1,[2,7]),(\mathrm{A} 2,[10,15]),(\mathrm{A} 5,[16,46]),(\mathrm{A} 6,[50,60]), \\
& \quad(\mathrm{A} 8,[200,210]),(\mathrm{A} 9,[211,241]),(\mathrm{A} 10,[211,291]),(\mathrm{A} 11,[311,411]), \\
& (\mathrm{A} 12,[412,612]),(\mathrm{A} 13,[613,628]),(\mathrm{A} 14,[629,634])\}
\end{aligned}
$$

\section{Abduction and Incomplete Processes}

Abduction is a non-monotonic reasoning process where hypotheses are made to explain observed facts [17]. While deductive reasoning focuses on deciding if a formula $\phi$ logically follows from a set $\Gamma$ of 
logical assertions known to hold, in abductive reasoning it is assumed that $\phi$ holds (as it corresponds to a set of observed facts) but it cannot be directly inferred by $\Gamma$. To make $\phi$ a consequence of $\Gamma$, abduction looks for a further set $\Delta$ of hypothesis, taken from a given set of abducible $\mathcal{A}$, which complements $\Gamma$ in such a way that $\phi$ can be inferred (in symbols $\Gamma \cup \Delta \models \phi$ ). The set $\Delta$ is called abductive explanation (of $\phi$ ). In addition, $\Delta$ must usually satisfy a set of (domain-dependent) integrity constraints $\mathcal{I C}$ (in symbols, $\Gamma \cup \Delta \models \mathcal{I C}$ ). A typical integrity constraint (IC) is a denial, which expresses that two explanations are mutually exclusive.

Abduction has been introduced in the framework of Logic Programming in [12]. There, an $A b-$ ductive Logic Program (ALP) is defined as a triple $\langle\Gamma, \mathcal{A}, \mathcal{I C}\rangle$, where: (i) $\Gamma$ is a logic program, (ii) $\mathcal{A}$ is a set of abducible predicates, and (iii) $\mathcal{I C}$ a set of ICs. Given a goal $\phi$, abductive reasoning looks for a set of literals $\Delta \subseteq \mathcal{A}$ such that they entail $\phi \cup \mathcal{I C}$. The integration of constraint solving in abductive logic programming (ACLP) [12, 13] enhances the practical utility of ALP by enriching the representation of the problem domain and by improving the computation of abductive explanations.

In this paper we leverage on ACLP and on the SCIFF abductive logic programming framework [14]. The latter is an extension of the IFF abductive proof procedure [18] which, beside the general notion of abducible, natively supports the key notions of happened event, expectation, and compliance of an observed execution with a set of expectations. This makes SCIFF a suitable framework for dealing with event log incompleteness. Let a be an event corresponding to the execution of process activities, and $T$ (possibly with subscripts) its execution time. Abducibles are used in this work to make hypothesis on events that are not recorded in the examined trace. They are denoted using $\mathbf{A B D}(\mathrm{a}, T)$. Happened events are non-abducible, and account for events that have been logged in the trace. They are denoted using $\mathbf{H}(\mathrm{a}, T)$. Expectations $\mathbf{E}(\mathrm{a}, T)$, instead, model events that should occur (and therefore should be present in a trace). Compliance is described in Section 3.2.

ICs in SCIFF are used to relate happened events / abduced predicates with expectations / predicates to be abduced. Specifically, an IC is a rule of the form body $\rightarrow$ head, where body contains a conjunction of happened events, general abducibles, and defined predicates, while head contains a disjunction of conjunctions of expectations, general abducibles, and defined predicates.

Example 3.1. The fact that whenever an order is paid, then a receipt has to be emitted within 24 time units at the latest, can be encoded in SCIFF as the following IC:

$$
\mathbf{H}\left(\text { pay-order, } T_{p}\right) \rightarrow \mathbf{E}\left(\text { emit-receipt, } T_{e}\right) \wedge T_{e}>T_{p} \wedge T_{e}<T_{p}+24
$$

\subsection{Encoding SPOTs in SCIFF}

We show how SPOTs and their traces can be encoded in SCIFF. We start by considering traces. Since SCIFF natively provides the notion of happened event, it also comes with a notion of trace.

\section{Definition 3.2. (SCIFF trace)}

A (SCIFF) trace is a set of terms of type $\mathbf{H}(e, t)$, where $e$ is a ground term describing the happened event, and $t \in \mathbb{N}$ is the time instant at which the event occur.

Differently from SPOT traces, SCIFF traces contain punctual and fully specified events only. 
Since well-formedness of SPOTs forbids the repetition of activities, compliant traces cannot contain multiple event occurrences referring to the same activity. Thanks to this property, we can directly encode a fully specified SPOT $\mathcal{T}$ into a corresponding SCIFF trace $\sigma_{\mathbf{H}}(\mathcal{T})$, by representing each entry $\left(\mathrm{a},\left[t_{s}, t_{e}\right]\right) \in \mathcal{T}$ using two distinct happened events, one for the event start, and one for the event completion. Specifically, the translation satisfies the following key property: $\left(\mathrm{a},\left[t_{s}, t_{e}\right]\right) \in \mathcal{T}$ if and only if $\left\{\mathbf{H}\left(\operatorname{start}(\mathrm{a}), t_{s}\right), \mathbf{H}\left(\operatorname{end}(\mathrm{a}), t_{e}\right)\right\} \subseteq \sigma_{\mathbf{H}}(\mathcal{T})$. Punctual events denoting the execution of atomic activities are instead directly mapped to a single happened event.

Example 3.3. Trace (1) is represented in SCIFF as:

$$
\{\mathbf{H}(\operatorname{start}(\mathrm{a} 1), 2), \mathbf{H}(\operatorname{end}(\mathrm{a} 1), 7), \mathbf{H}(\operatorname{start}(\mathrm{a} 2), 10), \mathbf{H}(\operatorname{end}(\mathrm{a} 2), 15), \ldots\}
$$

The encoding of a SPOT model $\mathcal{P}=\langle\mathcal{A}$, obs, $P$, dur, tcon $\rangle$ in SCIFF consists of a set $\mathcal{I C}_{\mathcal{P}}$ of integrity constraints that account for: $(i)$ the semantics of activity execution, considering every activity in $\mathcal{A}$ together with its observability (as defined by obs) and duration (as defined by dur); (ii) the semantics of the process over $\mathcal{A}$, as defined by $P$ and its inner blocks; (iii) the temporal constraints introduced by tcon. We review each such contribution next.

Semantics of activity execution. The semantics of activity execution relates the start event of each activity a in $\mathcal{A}$ with the corresponding end event. The nature of such relationship depends on the observability of a, as well as its duration. On the one hand, the observability determines whether the start/end events for a have to be found explicitly in the trace, or are instead hypothesized. In the first case, the abstractions of happened/expected event provided by SCIFF are employed; in the latter case, instead, a generic abducible ABD is used. On the other hand, the duration induces temporal constraints binding the timestamps of the start and end event.

Specifically, let $\operatorname{dur}(\mathrm{a})=\langle m, n\rangle$. If a is observable, the set $\mathcal{I C}_{\mathcal{P}}$ contains the following rules:

$$
\begin{aligned}
\mathbf{H}(\operatorname{start}(\mathrm{a}), T) \wedge \mathbf{H}\left(\operatorname{start}(\mathrm{a}), T_{2}\right) \wedge T_{2} \neq T & \rightarrow \perp \\
\mathbf{H}(\operatorname{end}(\mathrm{a}), T) \wedge \mathbf{H}\left(\operatorname{end}(\mathrm{a}), T_{2}\right) \wedge T_{2} \neq T & \rightarrow \perp \\
\mathbf{H}\left(\operatorname{start}(\mathrm{a}), T_{s}\right) & \rightarrow \mathbf{E}\left(\operatorname{end}(\mathrm{a}), T_{e}\right) \wedge T_{e}-T_{s} \geq m \wedge T_{e}-T_{s} \leq n \\
\mathbf{H}\left(\operatorname{end}(\mathrm{a}), T_{e}\right) & \rightarrow \mathbf{E}\left(\operatorname{start}(\mathrm{a}), T_{e}\right) \wedge T_{e}-T_{s} \geq m \wedge T_{e}-T_{s} \leq n(13
\end{aligned}
$$

where, in the case where $n=\infty$, constraint $T_{e} \leq T_{s}+n$ simply reduces to true.

Rules (10) and (11) express that every activity can be repeated at most once, in agreement with the notion of SPOT trace. Rules (12) and (13), relate the start and completion of each activity, stating that whenever the activity is started, it is expected to be completed at a time that is compatible with the duration of the activity, and vice-versa.

If $\mathrm{a}$ is unobservable, that is, $\operatorname{obs}(\mathrm{a})=\mathrm{n}$, the set $\mathcal{I} \mathcal{C}_{\mathcal{P}}$ contains a variant of the three integrity constraints above, where the generic abducible ABD substitutes $\mathbf{H}$ and $\mathbf{E}$ :

$$
\begin{gathered}
\mathbf{A B D}(\operatorname{start}(\mathrm{a}), T) \wedge \mathbf{A B D}\left(\operatorname{start}(\mathrm{a}), T_{2}\right) \wedge T_{2} \neq T \rightarrow \perp \\
\mathbf{A B D}(\text { end }(\mathrm{a}), T) \wedge \mathbf{A B D}\left(\operatorname{end}(\mathrm{a}), T_{2}\right) \wedge T_{2} \neq T \rightarrow \perp \\
\mathbf{A B D}\left(\operatorname{start}(\mathrm{a}), T_{s}\right) \rightarrow \mathbf{A B D}\left(\operatorname{end}(\mathrm{a}), T_{e}\right) \wedge T_{e}-T_{s} \geq m \wedge T_{e}-T_{s} \leq n \\
\mathbf{A B D}\left(\operatorname{end}(\mathrm{a}), T_{e}\right) \rightarrow \mathbf{A B D}\left(\operatorname{start}(\mathrm{a}), T_{s}\right) \wedge T_{e}-T_{s} \geq m \wedge T_{e}-T_{s} \leq n
\end{gathered}
$$


where, in the case where $n=\infty$, constraint $T_{e} \leq T_{s}+n$ simply reduces to true.

Finally, if a is partially observable, that is, obs $(\mathrm{a})=\mathrm{p}$, the formalization of the execution of a has to simultaneously account for the case where the execution of a is observed, as well that where the execution of a is not observed, and may consequently be hypothesized. This is done by imposing all integrity constraints $(10)-(16)$, together with the following ones, expressing that the execution of a may be either observed or hypothesized, but not both:

$$
\begin{array}{r}
\mathbf{H}\left(\operatorname{start}(\mathrm{a}), T_{s}\right) \wedge \mathbf{A B D}\left(\operatorname{start}(\mathrm{a}), T_{s}\right) \rightarrow \perp \\
\mathbf{H}\left(\operatorname{end}(\mathrm{a}), T_{s}\right) \wedge \mathbf{A B D}\left(\operatorname{end}(\mathrm{a}), T_{s}\right) \rightarrow \perp
\end{array}
$$

Encoding of the process. The process is encoded in SCIFF starting from the top block $P$, formalizing its execution semantics according to the definition of compliance (Definition 2.8), then proceeding recursively over its inner blocks. As for the encoding, we assume that $P$ and all its inner blocks are in normal form, and given a block $B$, we denote by in-B and out- $\mathrm{B}$ the events corresponding to the unobservable atomic tasks marking that the execution is respectively entering into and exiting from $B$. Technically, the set $\mathcal{I C}_{\mathcal{P}}$ of integrity constraints contains all constraints produced by the translation function $\tau$ applied to $P$, where $\tau$ is defined as follows. Notice that such constraints mirror in SCIFF the different aspects of Definition 2.11. In particular, each constraint is paired with one or (in the case of constraints with disjunctive heads) two corresponding constraints inverting the head and the body, thus realizing the if and only if semantics of Definition 2.8. As two generic rules that apply to each bock, we need to impose that the block is entered and exited only once. That is, for every block $B$ that is part of the SPOT of interest, we have:

$$
\begin{aligned}
& \mathbf{A B D}(\text { in- } \mathrm{B}, T) \wedge \mathbf{A B D}\left(\text { in- } \mathrm{B}, T_{2}\right) \wedge T_{2} \neq T \rightarrow \perp \\
& \mathbf{A B D}(\text { out-B, } T) \wedge \mathbf{A B D}\left(\text { out-B, } T_{2}\right) \wedge T_{2} \neq T \rightarrow \perp
\end{aligned}
$$

(Top block) Assuming $P=\left\langle\right.$ seq, $\left\langle\operatorname{in}_{P}, P^{\prime}\right.$, out $\left.\left.P\right\rangle\right\rangle$, we have that $\tau(P)=\{(22)\} \cup \tau\left(P^{\prime}\right)$, where:

$$
\text { true } \rightarrow \mathbf{A B D}(\text { in- } P, T)
$$

models that the process has to start, mirroring point (i) of Definition 2.11 .

(Task block - base case) Consider a block $B=\left\langle\right.$ seq, $\left\langle\right.$ in $_{B}$, a, out $\left.\left.{ }_{B}\right\rangle\right\rangle$, where a $\in \mathcal{A}$. Intuitively, the execution semantics of such a block states that whenever the block is entered, then a is expected to be started in the future, and that as soon as a is completed, the execution exits from the corresponding block. To formalize this intuition, we again have to proceed by cases, depending on the observability of a. If a is observable, $\tau(B)$ consists of:

$$
\begin{aligned}
\mathbf{A B D}\left(\text { in- } \mathrm{B}, T_{i n}\right) & \rightarrow \mathbf{E}\left(\text { start }(\mathrm{a}), T_{s}\right) \wedge T_{s} \geq T_{i n} \\
\mathbf{H}\left(\operatorname{start}(\mathrm{a}), T_{s}\right) & \rightarrow \mathbf{A B D}\left(\text { in- } \mathrm{B}, T_{i n}\right) \wedge T_{s} \geq T_{i n} \\
\mathbf{H}\left(\text { end }(\mathrm{a}), T_{e}\right) & \rightarrow \mathbf{A B D}\left(\text { out- } \mathrm{B}, T_{e}\right) \\
\mathbf{A B D}\left(\text { out-B }, T_{e}\right) & \rightarrow \mathbf{E}\left(\text { end }(\mathrm{a}), T_{e}\right)
\end{aligned}
$$


Together with constraints (10)-(13), constraints (23)-(26) mirror point 1 in Definition 2.8, as well as Definition 2.9. If a is unobservable, then its start/end are hypothesized, hence $\tau(B)$ becomes:

$$
\begin{aligned}
\mathbf{A B D}\left(\text { in- } \mathrm{B}, T_{i n}\right) & \rightarrow \mathbf{A B D}\left(\operatorname{start}(\mathrm{a}), T_{s}\right) \wedge T_{s} \geq T_{i n} \\
\mathbf{A B D}\left(\operatorname{start}(\mathrm{a}), T_{s}\right) & \rightarrow \mathbf{A B D}\left(\operatorname{in}-\mathrm{B}, T_{i n}\right) \wedge T_{s} \geq T_{i n} \\
\mathbf{A B D}\left(\operatorname{end}(\mathrm{a}), T_{e}\right) & \rightarrow \mathbf{A B D}\left(\text { out }-\mathrm{B}, T_{e}\right) \\
\mathbf{A B D}\left(\text { out-B, } T_{e}\right) & \rightarrow \mathbf{A B D}\left(\operatorname{end}(\mathrm{a}), T_{e}\right)
\end{aligned}
$$

Finally, if a is partially observable, the effect of entering into the block may be either that of expecting the start of a to occur in the trace, or to hypothesize it (cf. constraint (31) and its two "only if" constraints (32) and (33)). Specularly, the block is exited if and only if the completion of a is either observed or hypothesized, at the same time (cf. constraints (34)-(36)). Mutual exclusion between the case where the start (respectively, completion) of a is observed, and that where it is hypothesized, is guaranteed by the integrity constraint (18) (respectively, (19)). In formulae, $\tau(B)$ consists of:

$$
\begin{aligned}
& \mathbf{A B D}\left(\mathrm{in}-\mathrm{B}, T_{\text {in }}\right) \rightarrow \mathbf{E}\left(\operatorname{start}(\mathrm{a}), T_{s}\right) \wedge T_{s} \geq T_{\text {in }} \vee \mathbf{A B D}\left(\operatorname{start}(\mathrm{a}), T_{s}\right) \wedge T_{s} \geq T_{\text {in }} \\
& \mathbf{H}\left(\operatorname{start}(\mathrm{a}), T_{s}\right) \rightarrow \mathbf{A B D}\left(\text { in- } \mathrm{B}, T_{i n}\right) \wedge T_{s} \geq T_{\text {in }} \\
& \mathbf{A B D}\left(\operatorname{start}(\mathrm{a}), T_{s}\right) \rightarrow \mathbf{A B D}\left(\text { in- } \mathrm{B}, T_{\text {in }}\right) \wedge T_{s} \geq T_{\text {in }} \\
& \mathbf{H}\left(\operatorname{end}(\mathrm{a}), T_{e}\right) \rightarrow \mathbf{A B D}\left(\text { out-B }, T_{e}\right) \\
& \mathbf{A B D}\left(\text { end }(\mathrm{a}), T_{e}\right) \rightarrow \mathbf{A B D}\left(\text { out }-\mathrm{B}, T_{e}\right) \\
& \mathbf{A B D}\left(\text { out }-\mathrm{B}, T_{e}\right) \rightarrow \mathbf{E}\left(\text { end }(\mathrm{a}), T_{e}\right) \vee \mathbf{A B D}\left(\operatorname{end}(\mathrm{a}), T_{e}\right)
\end{aligned}
$$

(Inductive case - Sequence) Consider block $B$ of the form $\left\langle\right.$ seq, $\left\langle\mathrm{in}_{B},\left\langle\right.\right.$ seq, $\left.B_{1}, B_{2}\right\rangle$, out ou $\left.\left._{B}\right\rangle\right\rangle$. The encoding $\tau(B)$ of $B$ consists of a series of integrity constraints that chain the start/completion of the inner blocks. Specifically, $\tau(B)=\{(37)-42)\} \cup \tau\left(B_{1}\right) \cup \tau\left(B_{2}\right)$, where:

$$
\begin{aligned}
\mathbf{A B D}\left(\text { in- }^{-}, T_{\text {in }}\right) & \rightarrow \mathbf{A B D}\left(\text { in- }_{1}, T_{\text {in }}\right) \\
\mathbf{A B D}\left(\text { in- }_{1}, T_{\text {in }}\right) & \rightarrow \mathbf{A B D}\left(\text { in- } \mathrm{B}, T_{\text {in }}\right) \\
\mathbf{A B D}\left(\text { out }_{1}, T_{1}\right) & \rightarrow \mathbf{A B D}\left(\text { in- }_{2}, T_{1}\right) \\
\mathbf{A B D}\left(\text { in- }_{2}, T_{1}\right) & \rightarrow \mathbf{A B D}\left(\text { out }_{1}, T_{1}\right) \\
\mathbf{A B D}\left(\text { out- } \mathrm{B}_{2}, T_{\text {out }}\right) & \rightarrow \mathbf{A B D}\left(\text { out- }, T_{\text {out }}\right) \\
\mathbf{A B D}\left(\text { out-B }, T_{\text {out }}\right) & \rightarrow \mathbf{A B D}\left(\text { out- } \mathrm{B}_{2}, T_{\text {out }}\right)
\end{aligned}
$$

Constraints (37) and (38) model that as soon as block $B$ is entered, its first inner block in the sequence is entered, and vice-versa. Constraints (39) and (40) model that the execution exits from the first block of the sequence if and only if it enters into the consequent one. Finally, constraints (41) and (42) model that as soon as the execution exits from the second block of the sequence, it exits from the overall sequence block $B$, and vice-versa.

(Inductive case - Parallel split) Consider block $B$ of the form $\left\langle\right.$ and, $\left\langle\operatorname{in}_{B},\left\langle\right.\right.$ seq, $\left.B_{1}, B_{2}\right\rangle$, out $\left.\left.{ }_{B}\right\rangle\right\rangle$. The encoding $\tau(B)$ of $B$ captures the parallel execution of the two inner blocks, synchronizing upon their 
completion. Specifically, $\tau(B)=\{(43)-(48)\} \cup \tau\left(B_{1}\right) \cup \tau\left(B_{2}\right)$, where:

$$
\begin{aligned}
& \mathbf{A B D}\left(\text { in- } \mathrm{B}, T_{i n}\right) \rightarrow \mathbf{A B D}\left(\text { in- }_{1}, T_{i n}\right) \wedge \mathbf{A B D}\left(\text { in- }_{2}, T_{\text {in }}\right)(43) \\
& \mathbf{A B D}\left(\text { in }^{-B_{1}}, T_{i n}\right) \rightarrow \mathbf{A B D}\left(\text { in- } \mathrm{B}, T_{i n}\right) \\
& \mathbf{A B D}\left(\text { in }_{-} \mathrm{B}_{2}, T_{i n}\right) \rightarrow \mathbf{A B D}\left(\text { in- } \mathrm{B}, T_{i n}\right) \\
& \mathbf{A B D}\left(\text { out }-\mathrm{B}_{1}, T_{o 1}\right) \wedge \mathbf{A B D}\left(\text { out- } B_{2}, T_{o 2}\right) \wedge T_{o 1} \geq T_{o 2} \rightarrow \mathbf{A B D}\left(\text { out-B }, T_{o 1}\right) \\
& \mathbf{A B D}\left(\text { out }-\mathrm{B}_{1}, T_{o 1}\right) \wedge \mathbf{A B D}\left(\text { out- } B_{2}, T_{o 2}\right) \wedge T_{o 1}<T_{o 2} \rightarrow \mathbf{A B D}\left(\text { out-B }, T_{o 2}\right) \\
& \mathbf{A B D}\left(\text { out-B }, T_{o}\right) \rightarrow \mathbf{A B D}\left(\text { out- } B_{1}, T_{o}\right) \wedge \mathbf{A B D}\left(\text { out- } \mathrm{B}_{2}, T_{o 2}\right) \wedge T_{o} \geq T_{o 2} \\
& \vee \mathbf{A B D}\left(\text { out- } \mathrm{B}_{1}, T_{o 1}\right) \wedge \mathbf{A B D}\left(\text { out- } \mathrm{B}_{2}, T_{o}\right) \wedge T_{o} \geq T_{o 1}
\end{aligned}
$$

Rules (43)- (45) model that the execution enters $B$ if and only if it enters $B_{1}$ if and only if it enters $B_{2}$. Rules (46) and (47) model synchronization upon the completion of such sub-blocks, dictating that the execution exits from $B$ as soon as both $B_{1}$ and $B_{2}$ are completed, respectively handling the case where $B_{1}$ completes simultaneously/after or before $B_{2}$. Their converse constraint is captured by (48), stating that whenever $B$ completes, then its latest sub-block must complete at the same time.

(Inductive case - Exclusive choice) Consider block $B$ of the form $\left\langle\right.$ xor, $\left\langle\operatorname{in}_{B},\left\langle\right.\right.$ seq, $\left.B_{1}, B_{2}\right\rangle$, out $\left.\left.{ }_{B}\right\rangle\right\rangle$. The encoding $\tau(B)$ of $B$ captures the alternative execution of one of the two inner blocks. Specifically, $\tau(B)=\{49\}-55\} \cup \tau\left(B_{1}\right) \cup \tau\left(B_{2}\right)$, where:

$$
\begin{aligned}
& \mathbf{A B D}\left(\text { in- } \mathrm{B}, T_{i n}\right) \rightarrow \mathbf{A B D}\left(\text { in } \mathrm{B}_{1}, T_{\text {in }}\right) \vee \mathbf{A B D}\left(\text { in } \mathrm{B}_{2}, T_{\text {in }}\right) \\
& \mathbf{A B D}\left(\mathrm{in}^{\left.-\mathrm{B}_{1}, T_{i n}\right)} \rightarrow \mathbf{A B D}\left(\mathrm{in}-\mathrm{B}, T_{i n}\right)\right. \\
& \mathbf{A B D}\left(\text { in }^{-B_{2}}, T_{i n}\right) \rightarrow \mathbf{A B D}\left(\mathrm{in}-\mathrm{B}, T_{i n}\right) \\
& \mathbf{A B D}\left(\text { in }^{-B_{1}}, T_{\text {in }}\right) \wedge \mathbf{A B D}\left(\text { in- }_{2}, T_{\text {in }}\right) \rightarrow \perp \\
& \mathbf{A B D}\left(\text { out- } \mathrm{B}_{1}, T_{\text {out }}\right) \rightarrow \mathbf{A B D}\left(\text { out-B, } T_{\text {out }}\right) \\
& \mathbf{A B D}\left(\text { out- } B_{2}, T_{\text {out }}\right) \rightarrow \mathbf{A B D}\left(\text { out-B }, T_{\text {out }}\right) \\
& \mathbf{A B D}\left(\text { out-B }, T_{\text {out }}\right) \rightarrow \mathbf{A B D}\left(\text { out- } \mathrm{B}_{1}, T_{\text {out }}\right) \vee \mathbf{A B D}\left(\text { out- } \mathrm{B}_{2}, T_{\text {out }}\right)
\end{aligned}
$$

Constraints (49)-(52) model that as soon as the execution enters into $B$, it enters into exactly one block between $B_{1}$ and $B_{2}$, and vice-versa. Specularly, constraints (53) and (54) model that as soon as the execution exits from one of blocks $B_{1}$ or $B_{2}$, it simultaneously exists from the overall block $B$ as well. Conversely, constraint (55) captures if block $B$ completes, then one of its inner sub-blocks have to complete at the same time.

Encoding of inter-task constraints. Each inter-task constraint is directly encoded in SCIFF using a dedicated integrity constraints imposing that whenever the two events mentioned in the inter-task constraint occur, their corresponding execution time shall respect the imposed duration interval. Technically, let $e v: \mathcal{A} \times\{\mathfrak{s}, \mathfrak{e}\} \rightarrow \mathbb{S}$ be a total function that, given an activity and a start/end inscription, produces a string representing the corresponding event: for every a $\in \mathcal{A}$, we have $\operatorname{ev}(\mathrm{a}, \mathfrak{s})=\operatorname{start}(\mathrm{a})$, and $e v(\mathrm{a}, \mathfrak{e})=\operatorname{end}(\mathrm{a})$.

For every pair of activities $\mathrm{a}_{1}, \mathrm{a}_{2} \in \mathcal{A}$, and every pair of inscriptions $i_{1}, i_{2} \in\{\mathfrak{s}, \mathfrak{e}\}$ such that $\operatorname{tcon}\left(\mathrm{a}_{1}, i_{1}, \mathrm{a}_{2}, i_{2}\right)$ is defined and gives $\langle m, n\rangle$, set $\mathcal{I C}_{\mathcal{P}}$ contains a dedicated integrity constraint, 
whose exact shape depends on the observability of $a_{1}$ and $a_{2}$. If $a_{1}$ and $a_{2}$ are observable, we get:

$$
\mathbf{H}\left(e v\left(\mathrm{a}_{1}, i_{1}\right), T_{1}\right) \wedge \mathbf{H}\left(e v\left(\mathrm{a}_{2}, i_{2}\right), T_{2}\right) \rightarrow T_{2}-T_{1} \geq m \wedge T_{2}-T_{1} \leq n
$$

If $a_{1} / a_{2}$ is unobservable, the corresponding happened event is replaced by the generic abducible $\operatorname{ABD}\left(e v\left(\mathrm{a}_{1}, i_{1}\right), T_{1}\right) / \mathbf{H}\left(e v\left(\mathrm{a}_{2}, i_{2}\right), T_{2}\right)$. Finally, in the case of partial observability, both possibilities have to be considered, thus obtaining 2 to 4 distinct integrity constraints.

Example 3.4. The inter-task constraint between $A 8$ and $A 11$ in Figure 1 indicares that the elapsed time between the start of the scheduling of a surgery (A8), and the end of the surgery itself (A11), should be between 100 and 140 time units. Assuming that $A 8$ is observable while $A 11$ is partially observable, such inter-task constraint would be encoded in SCIFF using the following two rules:

$$
\begin{aligned}
\mathbf{H}\left(\operatorname{start}(\mathrm{a} 8), T_{8}\right) \wedge \mathbf{H}\left(\text { end }(\mathrm{a} 11), T_{11}\right) & \rightarrow \quad T_{11}-T_{8} \geq 100 \wedge T_{11}-T_{8} \leq 140 . \\
\mathbf{H}\left(\operatorname{start}(\mathrm{a} 8), T_{8}\right) \wedge \mathbf{A B D}\left(\text { end }(\mathrm{a} 11), T_{11}\right) & \rightarrow \quad T_{11}-T_{8} \geq 100 \wedge T_{11}-T_{8} \leq 140
\end{aligned}
$$

\subsection{Compliance in SCIFF: Declarative Semantics}

Besides the ability to capture different workflow constructs in a modular manner, the second important characteristic of our framework concerns the ability to represent the different forms of reasoning introduced in Section 2 in a uniform manner in terms of (strong or conditional) compliance.

Let $t_{c}$ be the current time of an ongoing execution trace $\mathcal{T}$. Depending on the choice of $t_{c}$, compliance of the trace $\mathcal{T}$ w.r.t, a process model $M$ can be used to simulate different types of reasoning as follows:

(1) if $t_{c}=0$, i.e. the observed trace is empty, then conditional compliance is used to hypothesise at least one possible execution. Thus, we obtain model consistency;

(2) if $t_{c}=t_{\text {final }}$, i.e., the execution has reached a final state, then we are in the case of a-posteriori compliance checking (declined in strong and conditional depending on whether the trace is complete or not);

(3) if $0<t_{c}<t_{\text {final }}$, we obtain run-time monitoring. In fact SCIFF can be used to check the (conditional) compliance of the executed part of the trace and make hypothesis on its future evolution. Adding further constraints (e.g., the minimization of overall execution time) enables SCIFF to provide also prediction/recommendation.

Hence, by providing a formal notion of strong and conditional compliance, we accomodate the reasoning tasks of Section 2 in the SCIFF setting. To make this approach operational for SPOTs, we simply take a normal SPOT $\mathcal{P}$ and translate it into the corresponding $\mathcal{S}_{\mathcal{P}}=\left\langle\emptyset, \mathcal{A}, \mathcal{I} \mathcal{C}_{\mathcal{P}}\right\rangle$, where: (i) the logic program of $\mathcal{S}_{\mathcal{P}}$ is empty, (ii) the set of abducible predicates is defined as $\mathcal{A}=\{\mathbf{A B D} / 2, \mathbf{E} / 2\}$, where $\mathbf{E} / 2$ predicates represent expectations, and $\mathbf{A B D} / 2$ are predicates that can be abduced/hypothesized; (iii) $\mathcal{I C}_{\mathcal{P}}$ is the set of integrity constraints obtained by the translation function defined in Section 3.1 .

To recall the formal notion of compliance in SCIFF, we first need to introduce what an abductive explanation is 6

\footnotetext{
${ }^{6}$ We do not consider the abductive goal, as it is not needed for our treatment.
} 


\section{Definition 3.5. (Abductive explanation $\Delta$ )}

Given a SCIFF specification $\mathcal{S}=\langle\mathcal{K B}, \mathcal{A}, \mathcal{I C}\rangle$ and a SCIFF trace $\mathcal{T}$, a set $\Delta \subseteq \mathcal{A}$ is an abductive explanation for $\langle\mathcal{S}, \mathcal{T}\rangle$ if and only if

$$
\operatorname{Comp}(\mathcal{K B} \cup \mathcal{T} \cup \Delta) \cup \mathrm{CET} \cup T_{\mathbb{N}} \models \mathcal{I C}
$$

where Comp is the (two-valued) completion of a theory [19], CET stands for Clark Equational Theory [20] and $T_{\mathbb{N}}$ is the CLP constraint theory [21] for integers.

The following definition fixes the semantics for observable events, and provides the basis for understanding the alignment of a trace with a process model.

\section{Definition 3.6. ( $\mathcal{T}$-Fulfilment w.r.t. timestamp $\left.t_{c}\right)$}

Given a trace $\mathcal{T}$, an abducible set $\Delta$ is trace-fulfilled w.r.t. $t_{c}$ if for every event $\mathrm{e} \in\{\Delta \cup \mathcal{T}\}$ and for each time $\mathrm{t} \leq t_{c}, \mathbf{E}(\mathrm{e}, \mathrm{t}) \in \Delta$ if and only if $\mathbf{H}(\mathrm{e}, \mathrm{t}) \in \mathcal{T}$.

The "only if" direction defines the semantics of expectation, indicating that an expectation is fulfilled when it finds the corresponding happening event in the trace. The "if" direction captures the prescriptive nature of process models, whose closed nature require that only expected events may happen. Notice that fulfilment is restricted to time instants prior to $t_{c}$, thus capturing the fact that the past (events before $t_{c}$ ) is somehow "closed", while the future is intrinsically open.

Given an abductive explanation $\Delta$, fulfilment acts as a compliance classifier, which separates the legal/correct execution traces with respect to $\Delta$ from the wrong ones.

\section{Definition 3.7. (Compliance)}

A trace $\mathcal{T}$ is compliant with a SCIFF specification $\mathcal{S}$ w.r.t. a time instant $t_{c}$ if there exists an abducible set $\Delta$ such that: (i) $\Delta$ is an abductive explanation for $\langle\mathcal{S}, \mathcal{T}\rangle$, and (ii) $\Delta$ is $\mathcal{T}$-fulfilled w.r.t. $t_{c}$. We say that $\mathcal{T}$ is compliant with $\mathcal{S}$ if $\mathcal{T}$ is compliant with $\mathcal{S}$ w.r.t. the maximum time instant mentioned in $\mathcal{T}$. In this case, we say that $\Delta$ is a compliance witness of $\mathcal{T}$ w.r.t. $\mathcal{S}$.

If no abductive explanation that is also $\mathcal{T}$-fulfilled can be found, then $\mathcal{T}$ is not compliant with the specification of interest. Contrariwise, the abductive explanation witnesses compliance.

In the case of SCIFF specifications encoding SPOTs, the presence or absence of ABD predicates in an abductive explanation intuitively discriminates between conditional and strong compliance. For convenience, given an abducible set $\Delta$ over $\mathbf{E}$ and ABD predicates, we denote by $\Delta_{\mathbf{E}}$ and $\Delta_{\mathbf{A B D}}$ the two parts of $\Delta$ respectively constituted by all $\mathbf{E}$ and $\mathbf{A B D}$ facts contained in $\Delta$. In addition, we assume, without loss of generality, that abducible facts only refer to activities and blocks present in the original SPOT of interest.

We now formally relate the notion of compliance for SPOTs, and the corresponding notion of compliance in SCIFF, consequently establishing that the encoding of SPOTs in SCIFF described in the previous section is actually correct, in the intuitive sense that it "preserves compliance".

For technical reasons, we need an encoding of a fully specified SPOT trace $\mathcal{T}$ that does not produce a corresponding SCIFF trace, but instead a set of $\mathbf{A B D} / \mathbf{E}$ facts respectively denoting the hypothetical and expected execution of its contained events. We respectively denote by $\sigma_{\mathbf{A B D}}(\mathcal{T})$ and $\sigma_{\mathbf{E}}(\mathcal{T})$ the encodings of $\mathcal{T}$ mirroring that of $\sigma_{\mathbf{H}}(\mathcal{T})$, but using $\mathbf{A B D} / \mathbf{E}$ predicates instead of $\mathbf{H}$ 
ones. We also consider the corresponding inverse encodings, mapping $\mathbf{H} / \mathbf{A B D} / \mathbf{E}$ facts back into corresponding events of a SPOT trace.

The following theorem establishes a close correspondence between the two notions of SPOT and SCIFF compliance, by considering fully specified traces. This is without loss of generality: partially specified traces are tackled by checking whether they admit at least one compliant attribute completion, for which then the theorem applies 7

Theorem 3.8. A fully specified logged trace $\mathcal{T}$ of a normal SPOT $\mathcal{P}$ is compliant with $\mathcal{P}$ if and only if $\sigma_{\mathbf{H}}(\mathcal{T})$ is compliant with $\mathcal{S}_{\mathcal{P}}$.

We prove a stronger version of Theorem 3.8 , captured by the following lemma.

Lemma 3.9. Let $\mathcal{P}=\langle\mathcal{A}$, obs, $P$, dur, tcon $\rangle$ be a normal SPOT, and let $\mathcal{T}$ be a fully specified logged trace of $\mathcal{P}$. The following two properties hold:

- (Completeness of the encoding) For every possible completion $\overline{\mathcal{T}}=\mathcal{T} \uplus \mathcal{T}_{\text {ext }}$ of $\mathcal{T}$ for $\mathcal{P}$ over $\mathcal{A}$, if $\overline{\mathcal{T}}$ is a compliance witness for $\mathcal{T}$ w.r.t. $\mathcal{P}$, then $\sigma_{\mathbf{E}}(\mathcal{T}) \uplus \sigma_{\mathbf{A B D}}\left(\mathcal{T}_{\text {ext }}\right)$ is a compliance witness of $\sigma_{\mathbf{H}}(\mathcal{T})$ w.r.t. $\mathcal{S}_{\mathcal{P}}$.

- (Soundness of the encoding) For every abducible set $\Delta$ over $\mathbf{E}$ and $\mathbf{A B D}$ predicates, if $\Delta$ is a compliance witness of $\sigma_{\mathbf{H}}(\mathcal{T})$ w.r.t. $\mathcal{S}_{\mathcal{P}}$, then $\mathcal{T} \cup \sigma_{\text {ABD }}^{-}\left(\Delta_{\mathbf{A B D}}\right)$ is a compliance witness of $\mathcal{T}$ w.r.t. $\mathcal{P}$.

\section{Proof:}

For the sake of clarity, throughout the proof we use notation $\mathcal{T}$ to denote a SPOT trace, and notation $\mathfrak{T}$ to denote a SCIFF trace. In addition, with a slight abuse of terminology, we say that an abducible set $\Delta$ witnesses compliance of a SCIFF trace $\mathfrak{T}$ w.r.t. a set of integrity constraints $\mathcal{I C}$, if it witnesses compliance with $\mathcal{S}_{\mathcal{P}}$ by considering only $\mathcal{I C}$ as integrity constraints. In addition, when assessing compliance of a SPOT trace (resp., SCIFF trace ) with a block (resp., the SCIFF integrity constraints encoding a block), it is enough to consider the portion of the trace that explicitly mentions events that are relevant for the block, where relevance is inductively defined as follows:

- an event is relevant for a task block if it refers to the activity of that task block;

- an event is relevant for a block if it denotes the entry/exit point of that block, or is relevant for one of its sub-blocks.

This can be easily shown by considering the definition of block compliance, and by considering the translation of blocks into SCIFF. Such a modularity is useful because it allows us to prove the claims of the theorem by induction on the structure of the process blocks, moving bottom-up from activities to the top block.

Let $\mathcal{P}=\langle\mathcal{A}$, obs, $P, d u r$, tcon $\rangle$, and $\mathcal{S}_{\mathcal{P}}=\left\langle\emptyset, \mathcal{A}, \mathcal{I C}_{\mathcal{P}}\right\rangle$. First of all, we observe that, by construction, for $\overline{\mathcal{T}}$ to be compliant with $\mathcal{S}_{\mathcal{P}}$, the following property holds: for every task a $\in \mathcal{A}$, $\mathbf{H}\left(\operatorname{start}(\mathrm{a}), t_{s}\right) \in \sigma_{\mathbf{H}}(\mathcal{T})$ if and only if $\mathbf{H}\left(\operatorname{end}(\mathrm{a}), t_{e}\right) \in \sigma_{\mathbf{H}}(\mathcal{T})$ for some $t_{s}, t_{e} \in \mathbb{N}$. This is guaranteed by integrity constraints $(10)-(13)$ for the case of observable tasks; unobservable and partially observable tasks enjoy the same property, as witnessed by integrity constraints (14)-(19).

\footnotetext{
${ }^{7}$ In Section 3.3 we discuss that partially specified traces are natively handled by the operational counterpart of SCIFF, without requiring an a-priori grounding.
} 
We separately consider block compliance, duration compliance, and inter-task compliance.

As for block compliance, we proceed bottom-up by induction on the structure of the blocks in $P$, recalling that, by hypothesis, $\mathcal{P}$ is normal. Consider a generic block $B=\left\langle\right.$ seq, $\left\langle\operatorname{in}_{B}, B^{\prime}\right.$, out $\left.\left._{B}\right\rangle\right\rangle$ in $P$.

Task block. The base case is the one where $B^{\prime}=$ a with a $\in \mathcal{A}$. We consider the case where a is non-atomic. The atomic case can be proven analogously.

First of all, we observe that, since activities do not repeat, the SCIFF integrity constraints determining compliance in this case are all groundings of rules $(10)-(19)$ and rules $(23)-(36)$, such that the activity is a and the block identifier is $B$. We denote by $\mathcal{I} C_{\text {task }}^{B}$ the so-obtained set of integrity constraints.

Let us first consider the completeness of the encoding. By reformulating Definition 2.12 (item 1), we have that $\overline{\mathcal{T}}$ complies with $B$ if one of the two conditions holds:

1. The portion of $\overline{\mathcal{T}}$ relevant to $B$ is $\emptyset$, i.e., $\mathcal{T}$ does not contain occurrences of a, and $\mathcal{T}_{\text {ext }}$ does not contain occurrences of $\operatorname{in}_{B}$ nor out ${ }_{B}$. This is preserved by the encoding, as $\emptyset$ is a compliance witness of the empty trace (and, in turn, the whole $\sigma_{\mathbf{H}}(\mathcal{T})$ ) w.r.t. $\mathcal{I C}_{\text {task }}^{B}$ in the case where no occurrence of a appear in $\sigma_{\mathbf{H}}(\mathcal{T})$. This can be easily seen by inspecting constraints $\mathcal{I C}_{\text {task }}^{B}$ : a way to comply with those constraints is to vacuously satisfy them (i.e., avoid matching with their antecedents).

2. The portion of $\overline{\mathcal{T}}$ relevant to $B$ has the form $\overline{\mathcal{T}}_{\mathrm{a}}=\left\{\left(\mathrm{a},\left[t_{s}, t_{e}\right]\right),\left(\mathrm{in}_{B}, t_{i}\right),\left(\right.\right.$ out $\left._{B}, t_{o}\right)$ $t_{i}, t_{s}, t_{e}, t_{o} \in \mathbb{N}$ s.t. $\left.t_{i} \leq t_{s}<t_{e}=t_{o}\right\}$, i.e., there exists exactly a single occurrence of a in $\overline{\mathcal{T}}$ of the form $\left(\mathrm{a},\left[t_{s}, t_{e}\right]\right)$, and exactly one occurrence of $\mathrm{in}_{B}$ and one of out $\mathrm{t}_{B}$ in $\mathcal{T}_{\text {ext }}$, respectively of the form $\left(\operatorname{in}_{B}, t_{i}\right)$ and $\left(\right.$ out $\left._{B}, t_{o}\right)$, so that $t_{i} \leq t_{s}<t_{e}=t_{o}$. To show that compliance preserved by the encoding, we have to distinguish between the case where a actually occurs in $\mathcal{T}$, which in turn reflects that a is either observable or it is partially observable and its execution has been logged, from the case where a does not occur in $\mathcal{T}$ (and, consequently, occurs in $\mathcal{T}_{\text {ext }}$ ), which in turn reflects that a is either unobservable or it is partially observable and its execution has not been logged.

In the first case, by considering the relevant portion of $\sigma_{\mathbf{H}}(\mathcal{T})$ for $\mathcal{I C}_{\text {task }}^{B}$, we obtain the corresponding SCIFF trace $\mathfrak{T}_{\mathrm{a}}=\sigma_{\mathbf{H}}\left(\overline{\mathcal{T}}_{\mathrm{a}} \cap \mathcal{T}\right)=\left\{\mathbf{H}\left(\operatorname{start}(\mathrm{a}), t_{s}\right), \mathbf{H}\left(\operatorname{end}(\mathrm{a}), t_{e}\right) \mid t_{s}<\right.$ $\left.t_{e}\right\}$. and the corresponding abducible set $\Delta_{\mathrm{a}}=\sigma_{\mathbf{E}}\left(\overline{\mathcal{T}}_{\mathrm{a}} \cap \mathcal{T}\right) \cup \sigma_{\mathbf{A B D}}\left(\overline{\mathcal{T}}_{\mathrm{a}} \cap \mathcal{T}_{\text {ext }}\right)=$ $\left\{\mathbf{A B D}\left(\operatorname{in}_{B}, t_{i}\right), \mathbf{E}\left(\operatorname{start}(\mathrm{a}), t_{s}\right), \mathbf{E}\left(\operatorname{end}(\mathrm{a}), t_{e}\right), \mathbf{A B D}\left(\right.\right.$ out $\left.\left._{B}, t_{o}\right) \mid t_{i} \leq t_{s}<t_{e}=t_{o}\right\}$. It is then easy to show that $\Delta_{a}$ is a compliance witness of $\mathfrak{T}_{a}$ w.r.t. $\mathcal{I} \mathcal{C}_{\text {task }}^{B}$. Specifically:

- Rules (10) and (11) are satisfied (only one occurrence of a appears in $\mathfrak{T}_{\mathrm{a}}$ and, in turn, in the whole $\left.\sigma_{\mathbf{H}}(\overline{\mathcal{T}})\right)$.

- Rules (12), 13) are satisfied thanks to the ordered presence of the start and completion of a in $\mathfrak{T}_{\mathrm{a}}$; notice that the more specific temporal constraints related to a's duration will be discussed later on in the proof.

- Rules (14) - 17), as well as 27) - 30, are not part of $\mathcal{I C}_{\text {task }}^{B}$ in this case, since, by hypothesis, a is not unobservable.

- Rules (18) and (19) are satisfied, since the start and completion of a appear in $\mathfrak{T}_{\mathrm{a}}$, but their hypothetical execution does not appear in $\Delta_{\mathrm{a}}$.

- If a is observable, rules (23) - 26) are satisfied, as it can be straightforwardly seen by the shape of $\mathfrak{T}_{a}$ and that of $\Delta_{a}$. If $a$ is instead partially observable, rules 31 - (36) behave 
exactly as 23 - 26) (in particular, rules (31) and 36 are satisfied by $\mathfrak{T}_{\mathrm{a}} \cup \Delta_{\mathrm{a}}$, considering their first disjunct in the rule heads).

In the second case, since a is not present in $\mathcal{T}$, we simply get that the relevant portion of $\sigma_{\mathbf{H}}(\mathcal{T})$ for $\mathcal{I C}_{\text {task }}^{B}$ is the empty trace, while the corresponding abducible set becomes then $\Delta_{a}=$ $\sigma_{\mathbf{A B D}}\left(\overline{\mathcal{T}}_{\mathrm{a}} \cap \mathcal{T}_{\text {ext }}\right)=\left\{\mathbf{A B D}\left(\mathrm{in}_{B}, t_{i}\right), \mathbf{A B D}\left(\operatorname{start}(\mathrm{a}), t_{s}\right), \mathbf{A B D}\left(\operatorname{end}(\mathrm{a}), t_{e}\right), \mathbf{A B D}\left(\right.\right.$ out $\left._{B}, t_{o}\right), \mid$ $\left.t_{i} \leq t_{s}<t_{e}=t_{o}\right\}$. It is then easy to show that $\Delta_{\mathrm{a}}$ is a compliance witness of $\mathfrak{T}_{\mathrm{a}}$ w.r.t. $\mathcal{I C}_{\text {task }}^{B}$. Specifically:

- Rules $(10)-(13)$ are all vacuously satisfied, since no occurrence of a is contained in $\sigma_{\mathbf{H}}(\mathcal{T})$.

- Rules (14) - (17) are satisfied, since only one hypothetical occurrence of start(a) and of end (a) is contained in $\Delta_{a}$, with the right timestamp ordering.

- Rules (18) and (19) are satisfied, since the start and completion of a does not appear in $\sigma_{\mathbf{H}}(\mathcal{T})$.

- Rules (23)-26) are not part of $\mathcal{I C}_{\text {task }}^{B}$ since, by hypothesis, a is not fully observable.

- If a is unobservable, rules 27) - 30 are satisfied, as it can be straightforwardly seen by the shape of $\Delta_{a}$. If a is instead partially observable, rules $(31)$ - (36) behave exactly as $(27)-$ (30) (in particular, rules (31) and (36) are satisfied by $\Delta_{a}$, considering their second disjunct in the rule heads).

Let us now consider the soundness of the encoding. Two cases may arise: a occurs in $\mathcal{T}$, or it does not. If $\left(\mathrm{a},\left[t_{s}, t_{e}\right]\right) \in \mathcal{T}$, which is only possible if a is either observable or partially observable and it has been actually logged, then the only abducible set witnessing compliance of $\sigma_{\mathbf{H}}(\mathcal{T})$ w.r.t. $\mathcal{I C}_{\text {task }}^{B}$ has the form $\Delta_{\text {exec }}^{\mathrm{a}}=\Delta_{\text {exec }}^{\text {rest }} \uplus\left\{\mathbf{A B D}\left(\mathrm{in}_{B}, t_{i}\right), \mathbf{E}\left(\operatorname{start}(\mathrm{a}), t_{s}\right), \mathbf{E}\left(\operatorname{end}(\mathrm{a}), t_{e}\right), \mathbf{A B D}\left(\right.\right.$ out $\left._{B}, t_{o}\right) \mid t_{i} \leq$ $\left.t_{s}<t_{e}=t_{o}\right\}$, where $\Delta_{\text {exec }}^{\text {rest }}$ does not refer to activity a nor block $B$. To prove soundness, we can then abstract away from $\Delta_{\text {exec }}^{\text {rest }}$, and have to show that SPOT trace $\overline{\mathcal{T}}_{\mathrm{a}}=\left\{\left(\mathrm{a},\left[t_{s}, t_{e}\right]\right)\right\} \cup \sigma_{\mathbf{A B D}}^{-}\left(\Delta_{\text {exec }}^{\mathrm{a}}\right)=$ $\left\{\left(\mathrm{in}_{B}, t_{i}\right),\left(\mathrm{a},\left[t_{s}, t_{e}\right]\right),\left(\right.\right.$ out $\left.\left._{B}, t_{o}\right) \mid t_{i} \leq t_{s}<t_{e}=t_{o}\right\}$ is a compliance witness for $\left\{\left(\mathrm{a},\left[t_{s}, t_{e}\right]\right)\right\}$ w.r.t. $B$. This directly follows from Definition 2.8 (item 1 ).

If instead a does not occur in $\mathcal{T}$, two possible abducible sets witnessing compliance of $\emptyset$ w.r.t. $\mathcal{I C}_{\text {task }}^{B}$ do exist, considering that they cannot contain any expectation regarding a, as it would be violated by $\sigma_{\mathbf{H}}(\mathcal{T})$. In particular, the two possible compliance witnesses are

1. The empty abducible set (obtained by vacuous satisfaction of all the involved integrity constraints). This transfers back to SPOT as well, as argued above for the completeness proof.

2. Abducible set $\Delta_{h y p}^{\mathrm{a}}=\Delta_{\text {hyp }}^{\text {rest }} \uplus\left\{\mathbf{A B D}\left(\mathrm{in}_{B}, t_{i}\right), \mathbf{A B D}\left(\operatorname{start}(\mathrm{a}), t_{s}\right), \mathbf{A B D}\left(\mathrm{end}(\mathrm{a}), t_{e}\right)\right.$, $\mathbf{A B D}\left(\right.$ out $\left.\left._{B}, t_{o}\right) \mid t_{i} \leq t_{s}<t_{e}=t_{o}\right\}$, where $\Delta_{h y p}^{r e s t}$ does not refer to activity a nor block $B$. This is obtained in the case where a is not observable (and, hence, rules 27) - 30 are in place), or in the case where a is partially observable and not logged (and, hence, rules (31) $-(36)$, considering the second disjunct in rules (31) and (36)). To prove soundness, we can then abstract away from $\Delta_{\text {exec }}^{\text {hyp }}$, and have to show that the SPOT trace $\emptyset \cup \sigma_{\mathbf{A B D}}^{-}\left(\Delta_{h y p}^{\mathrm{a}}\right)=$ $\left\{\left(\right.\right.$ in $\left._{B}, t_{i}\right),\left(\mathrm{a},\left[t_{s}, t_{e}\right]\right),\left(\right.$ out $\left.\left._{B}, t_{o}\right) \mid t_{i} \leq t_{s}<t_{e}=t_{o}\right\}$ is a compliance witness for $\left\{\left(\mathrm{a},\left[t_{s}, t_{e}\right]\right)\right\}$ w.r.t. $B$. This has been already proved above.

Sequence block. Let $B^{\prime}=\left\langle\right.$ seq, $\left.B_{1}, B_{2}\right\rangle$. By induction hypothesis, we assume that $\overline{\mathcal{T}}$ is a compliance witness for $B_{1}$ and $B_{2}$. We denote by $\overline{\mathcal{T}}_{B_{1}}$ and $\overline{\mathcal{T}}_{B_{2}}$ the two (disjoint) portions of $\overline{\mathcal{T}}$ that are respectively relevant for $B_{1}$ and $B_{2}$, and by $\overline{\mathcal{T}}_{B}$ the portion of $\overline{\mathcal{T}}$ that is relevant for $B$. Notice that, by definition or relevance, $\overline{\mathcal{T}}_{B_{1}} \uplus \overline{\mathcal{T}}_{B_{2}} \subseteq \overline{\mathcal{T}}_{B}$. In addition, we denote by $\mathcal{I C}_{\text {seq }}^{B}=\mathcal{I C}_{B_{1}} \uplus \mathcal{I C}_{B_{2}} \uplus \mathcal{I C} \mathcal{C}_{B}$ the integrity constraints obtained from the encoding of $B_{1}$ and $B_{2}$, and the integrity constraints $\mathcal{I} \mathcal{C}_{B}$ 
relevant for $B$, which correspond all groundings of rules (37)-42), considering $B, B_{1}$, and $B_{2}$ as block identifiers.

Let us first consider the completeness of the encoding. By reformulating Definition 2.12 (item 2), we have that $\overline{\mathcal{T}}$ complies with $B$ if one of the two conditions holds:

1. The portion of $\overline{\mathcal{T}}$ relevant to $B$ coincides with $\overline{\mathcal{T}}_{B_{1}} \uplus \overline{\mathcal{T}}_{B_{2}}=\emptyset$. This means that the tace does not enter the block at all, nor its sub-blocks $B_{1}$ and $B_{2}$. This, in turn, means that that also the portions of $\overline{\mathcal{T}}$ relevant to $B_{1}$ and $B_{2}$ actually coincide with the empty trace. A property of all the integrity constraints encoding non-top blocks, is that the empty abducible set is a compliance witness for the empty trace w.r.t. such constraints. This clearly applies to $B_{1}$ and $B_{2}$ (which cannot be the top block), and immediately implies the claim to be proved, namely that the empty abducible set is an compliance witness for the empty trace w.r.t. $\mathcal{I C}_{\text {seq }}^{B}$.

2. The portion of $\overline{\mathcal{T}}$ relevant to $B$ has the form $\overline{\mathcal{T}}_{\text {seq }}=\overline{\mathcal{T}}_{B_{1}} \uplus \overline{\mathcal{T}}_{B_{2}} \uplus \overline{\mathcal{T}}_{B}$ with $\overline{\mathcal{T}}_{B}=$ $\left\{\left(\right.\right.$ in $\left._{B}, t_{i}\right),\left(\right.$ out $\left.\left._{B}, t_{o}\right)\right\}$, and satisfying the following conditions: $(i)$ there exists exactly one occurrence of $\operatorname{in}_{B_{1}}$ and of out $B_{1}$ in $\overline{\mathcal{T}}_{B_{1}}$, respectively of the form $\left(\mathrm{in}_{B_{1}}, t_{i 1}\right)$ and $\left(\right.$ out $\left._{B_{1}}, t_{o 1}\right)$; (ii) there exists exactly one occurrence of $\operatorname{in}_{B_{2}}$ and of out $_{B_{2}}$ in $\overline{\mathcal{T}}_{B_{2}}$, respectively of the form $\left(\right.$ in $\left._{B_{2}}, t_{i 2}\right)$ and $\left(\right.$ out $\left._{B_{2}}, t_{o 2}\right)$; (iii) the timestamps are so that $t_{i}=t_{i 1} \leq t_{o 1}=t_{i 2} \leq t_{o 2}=t_{o}$. Let $\mathfrak{T}_{\text {seq }}=\sigma_{\mathbf{H}}\left(\overline{\mathcal{T}}_{\text {seq }} \cap \mathcal{T}\right)$ be the relevant portion of $\sigma_{\mathbf{H}}(\mathcal{T})$ for $\mathcal{I C}_{\text {seq }}^{B}$, and let $\Delta_{\text {seq }}=$ $\sigma_{\mathbf{E}}\left(\overline{\mathcal{T}}_{\text {seq }} \cap \mathcal{T}\right) \cup \sigma_{\mathbf{A B D}}\left(\overline{\mathcal{T}}_{\text {seq }} \cap \mathcal{T}_{\text {ext }}\right)$ be the abducible set obtained from $\overline{\mathcal{T}}_{\text {seq }}$. In addition, for $i \in\{1,2\}$, let $\Delta_{B_{i}}=\sigma_{\mathbf{E}}\left(\overline{\mathcal{T}}_{B_{i}} \cap \mathcal{T}\right) \cup \sigma_{\mathbf{A B D}}\left(\overline{\mathcal{T}}_{B_{i}} \cap \mathcal{T}_{\text {ext }}\right)$. By induction hypothesis, we have that $\Delta_{B_{i}}$ is a compliance witness for $\overline{\mathcal{T}}_{B_{i}}$ w.r.t. $\mathcal{I} \mathcal{C}_{B_{i}}$. Note that, by construction, $\Delta_{B_{i}} \subseteq \Delta_{\text {seq }}$, and due to non-repetition of blocks and activities, $\Delta_{\text {seq }} \backslash \Delta_{B_{i}}$ does not refer to blocks and activities contained in $B_{i}$. For this reason, we can generalize the induction hypothesis and say that $\Delta_{\text {seq }}$ is a compliance witness for $\overline{\mathcal{T}}_{\text {seq }}$ w.r.t. $\mathcal{I} \mathcal{C}_{B_{i}}$. It then remains to only show that $\Delta_{\text {seq }}$ is a compliance witness for $\overline{\mathcal{T}}_{\text {seq }}$ w.r.t. $\mathcal{I C}_{B}$.

To this end, we first notice that the portion of $\overline{\mathcal{T}}_{\text {seq }}$ that interacts with $\mathcal{I C}_{B}$ has the form $\left\{\left(\right.\right.$ in $\left._{B}, t_{i}\right),\left(\right.$ in $\left._{B_{1}}, t_{i 1}\right),\left(\right.$ out $\left._{B_{1}}, t_{o 1}\right),\left(\right.$ in $\left._{B_{2}}, t_{i 2}\right),\left(\right.$ out $\left._{B_{2}}, t_{o 2}\right),\left(\right.$ out $\left._{B}, t_{o}\right) \mid t_{i}=t_{i 1} \leq t_{o 1}=$ $t_{i 2} \leq t_{o 2}=t_{o}$, which results in an empty SCIFF trace, and in the following abducible set $\Delta_{\text {rel }}=\left\{\mathbf{A B D}\left(\mathrm{in}_{B}, t_{i}\right), \mathbf{A B D}\left(\mathrm{in}_{B_{1}}, t_{i 1}\right), \mathbf{A B D}\left(\right.\right.$ out $\left._{B_{1}}, t_{o 1}\right), \mathbf{A B D}\left(\right.$ in $\left._{B_{2}}, t_{i 2}\right), \mathbf{A B D}\left(\right.$ out $\left._{B_{2}}, t_{o 2}\right)$, $\operatorname{ABD}\left(\right.$ out $\left.\left._{B}, t_{o}\right) \mid t_{i}=t_{i 1} \leq t_{o 1}=t_{i 2} \leq t_{o 2}=t_{o}\right\}$. It is then straightforward to see that $\Delta_{\text {rel }}$ is a compliance witness of $\emptyset$ w.r.t. $\mathcal{I C}_{B}$. By observing that:

- rules in $\mathcal{I C}_{B}$ do not mention any happened event, and

- due to non-repetition of blocks and activities, $\Delta_{B} \backslash \Delta_{\text {rel }}$ does not interact with the rules in $\mathcal{I C}_{B}$,

we consequently obtain that $\Delta_{\text {seq }}$ is a compliance witness of $\mathfrak{T}_{s e q}$ w.r.t. $\mathcal{I C}_{B}$.

We now turn to soundness of the encoding of a sequence block. By induction hypothesis, we have that $\sigma_{\mathbf{H}}(\mathcal{T})$ is compliant with $\mathcal{I C}_{B_{1}} \uplus \mathcal{I C}_{B_{2}}$. Specifically, for $i \in\{1,2\}$, there is an aducible set $\Delta_{B_{1}}$ that is a compliance witness of $\sigma_{\mathbf{H}}\left(\mathcal{T}_{B_{i}}\right)$ w.r.t. $\mathcal{I} \mathcal{C}_{B_{i}}$, where $\mathcal{T}_{B_{i}}$ is the portion of $\mathcal{T}$ that is relevant to block $B_{i}$. By considering the rules in $\mathcal{I C}_{\text {block }}$, for an abductive explanation $\Delta_{\text {seq }}^{B}$ to be a compliant witness of $\sigma_{\mathbf{H}}(\mathcal{T})$ w.r.t. $\mathcal{I} \mathcal{C}_{\text {block }}$, two situations may arise:

1. $\Delta_{\text {seq }}^{B}$ does not contain events referring to blocks $B, B_{1}, B_{2}$, and $\sigma_{\mathbf{H}}(\mathcal{T})$ does not contain events referring to activities that are (indirectly) contained in $B$, i.e., $\sigma_{\mathbf{H}}\left(\mathcal{T}_{B_{1}}\right)=\sigma_{\mathbf{H}}\left(\mathcal{T}_{B_{2}}\right)=\emptyset$. The claim then amounts to prove that the empty SPOT trace is compliant with a sequence block, 
which is clearly the case by considering Definition 2.12 (item 2).

2. $\Delta_{\text {seq }}^{B}=\Delta_{\text {rest }} \uplus \Delta_{B_{1}} \uplus \Delta_{B_{2}} \uplus \Delta_{B}$, where:

- $\Delta_{\text {rest }}$ does not contain any abducible fact referring to blocks and activities that are (indirectly) contained in $B$.

- For $i \in\{1,2\}, \Delta_{B_{1}}$ is a compliance witness of $\sigma_{\mathbf{H}}(\mathcal{T})$ w.r.t. $\mathcal{I C}_{B_{i}}$, and contains $\left\{\mathbf{A B D}\left(\right.\right.$ in $_{B_{i}}, t_{i i}, \mathbf{A B D}$ (out $\left._{B_{i}}, t_{o i} \mid t_{o i} \geq t_{i i}\right\}$. The fact that $t_{o i} \geq t_{i i}$ derives from the fact that every block (indirectly) contains one or more activities, which in turn require the exit from the block to appear after entering into the block.

- $\Delta_{B}=\left\{\mathbf{A B D}\left(\right.\right.$ in $_{B}, t_{i}, \mathbf{A B D}\left(\right.$ out $\left._{B}, t_{o} \mid t_{o} \geq t_{i}\right\}$.

- The timestamps of such abducible facts are so that $t_{i}=t_{i 1}$ (cf. rules (37) and (38)), $t_{o 1}=t_{i 2}$ (cf. rules (39) and (40)), and $t_{o 2}=t_{o}$ (cf. rules (41) and (42)).

Thus, we can abstract away from $\Delta_{\text {rest }}$, and we have to show that the SPOT trace $\mathcal{T}_{B_{1}} \uplus \mathcal{T}_{B_{2}} \uplus$ $\sigma_{\text {ABD }}^{-}\left(\Delta_{B_{1}} \uplus \Delta_{B_{2}} \uplus \Delta_{B}\right)$ is a compliance witness for $\mathcal{T}_{B_{1}} \uplus \mathcal{T}_{B_{2}}$ w.r.t. block $B$. After having applied the induction hypothesis, we have then to show that $\sigma_{\mathbf{A B D}}^{-}\left(\Delta_{B_{1}} \uplus \Delta_{B_{2}} \Delta_{B}\right)$ satisfies point 2.iv of Definition 2.12. This is straightforward by comparing the conditions imposed in such a point, and the construction of $\Delta_{B_{1}} \uplus \Delta_{B_{2}} \Delta_{B}$ detailed above.

Parallel split block. The proof is analogous to that of sequence, with the only difference in the temporal conditions attached to the block-related events. Such conditions, formalized in item 3 of Definition 2.12, are perfectly mirrored by integrity constraints (43)-(48).

Exclusive choice block. The preservation of temporal conditions proceeds analogoulsy to the other cases. The only interesting part is about mutual exclusion, that is, guaranteeing that it is not possible to produce a compliant witness in the case where both sub-blocks are executed. In the SPOT case, this is captured explicitly in point $4 . i v$ of Definition 2.12. In the SCIFF case, this is captured by integrity constraint [52].

Top block. The only peculiar aspect of the top block is that it must be entered. Every completion of $\mathcal{T}$ that witnesses compliance of $\mathcal{T}$ w.r.t. $\mathcal{P}$ contains an event of the form $\left(\operatorname{in}_{P}, t\right)$ for some $t \in \mathbb{N}$, as dictated by Definition 2.13 , item (iii). This is perfectly mirrored by the SCIFF encoding: every abducible set witnessing compliance of $\sigma_{\mathbf{H}}(\mathcal{T})$ w.r.t. $\mathcal{S}_{\mathcal{P}}$ must contain a fact of the form $\mathbf{A B D}\left(\operatorname{in}_{P}, t\right)$, as imposed by the integrity constraint (22).

We now tackle duration and inter-task compliance. Here, the proof is directly obtained by respectively comparing:

- Definition 2.9 with integrity constraints (12), (13), (16), (17);

- Definition 2.10 with integrity constraint (56).

Since the imposed temporal conditions coincide, every compliant SPOT trace completion imposes temporal conditions that are compatible with what the integrity constraints dictate. On the other hand, every SCIFF abductive explanation witnessing compliance must obey to the temporal conditions imposed by rules (12), (13), (16, , 17), 56, which in fact unconditionally apply to every activity-related event, and such conditions mimic what is expected by Definition 2.9 and efinition 2.10. This concludes the proof.

We conclude this section by recalling that the SCIFF proof procedure, which can be actually employed as a reasoning machinery on top of SCIFF specifications, has been proven sound and com- 
plete w.r.t. the SCIFF declarative semantics [14]. Our declarative semantics restricts the notions of fulfilment and compliance to a specific current time $t_{c}$, i.e., to open traces: hence soundness and completeness still hold [22], and can be directly applied to the case of monitoring as well. Furthermore, when reasoning over SCIFF specifications encoding SPOTs, it is guaranteed that the size of actual/hypothesized traces is bounded by the number of blocks and activities present in the SPOT under study. This, in turn, implies termination. In summary, we obtain that the SCIFF proof procedure can be effectively employed to reason on conditional/strong compliance of SPOTs either at run-time or a posteriori, obtaining correct answers in a finite amount of time. This is the subject of the next section.

\subsection{SCIFF at work}

The SCIFF proof procedure can be queried by specifying a goal. While the query is goal driven and supports a backward reasoning style, the use of integrity constraints allows to support a forward reasoning style at the same time. In the following, we use in- $B_{0}$ and out- $B_{0}$ to refer to the start and completion of the top block of the (normal) SPOT of interest.

In our approach the goal is always to prove $\mathbf{A B D}$ (in- $\left.\mathrm{B}_{0}, 0\right)$. The goal itself can be satisfied immediately, since start can be abduced (and added to the answer $\Delta$ ). However, abducing start will trigger an integrity constraint. For example, consider the normalized version of the FF process fragment in Example 2.3. In this case, in- $B_{0}$ is related to the sequence between the two task blocks for $A 1$ and A2, encoded through the instantiation of integrity constraints (37)-42 to the actual block names of the sequence. Since ABD(in- $\left.B_{0}, 0\right)$ appears in the body of (the instantiation of) constraint (37), the constraint triggers, generating the hypothesis that the first, inner task block is entered. This triggers the instantiation of constraint 23 , generating an expectation about the future start of $A 1$. From that, SCIFF either matches the expectation with the happening of $A 1$, or hypothesises it (depending on the observability of $A 1)$. In turn, this triggers other integrity constraints.

The proof procedure stops when no more integrity constraints can be triggered, and our encoding ensures that this happens only when the final activity out- $\mathrm{B}_{0}$ is reached. The outcome is the set $\Delta$ of hypotheses under which the goal is true, and all the ICs are satisfied. This, in the context of SPOT, corresponds to the compliance witness.

Notice that, operationally, the abductive answer $\Delta$ might contain abducibles with variables representing time instants, and this needs to be taken care of. In fact, we exploit ACLP to support temporal constraints among activity executions: a network of CLP constraints is used to restrict variables representing happening times to specific domains. SCIFF authors report that their proof procedure is complete, provided the underlying CLP solver is complete as well. In our case the CLP solver is not complete, unless an explicit assignment of a value to all the variables is requested through a so-called labelling (grounding) step [14]. As a consequence we may have an ALP solution $\Delta$, that would be made infeasible by the temporal constraints. To rule out this case, we explicitly require labelling, when needed.

In a similar way, the SCIFF proof procedure also supports natively the notion of partially specified trace, that is, happened events that contain variables, in turn explicitly accounting for incompleteness, as in a partially specified SPOT trace. This means that, when reasoning on partially specified SPOT traces, it is not required to ground them a-priori, but it is instead possible of encoding them into 
corresponding partially specified SCIFF traces. The proof procedure takes then care of attaching constraints and/or labeling, the variables contained therein.

Given a trace, and a workflow encoded as described in Section 3.1, the SCIFF proof procedure can be used to provide the different reasoning services introduced in Section 2. A first general observation is that all reasoning tasks may require labelling of temporal-related variables. Model consistency is directly supported by the SCIFF when the execution trace is empty. However, all the activities in the workflow must be partially observable or non-observable, so that the proof procedure can make hypotheses. This proviso also holds for runtime monitoring. Strong compliance corresponds to the original reasoning task of the SCIFF, and it is still supported in our context. Conditional compliance is supported thanks to our proposed mapping, where hypotheses about non-observed events are directly supported by the integrity constraints. Note that, depending on the completeness of the CLP solver used by SCIFF, non compliances due to temporal constraints might be detected as soon as a constraint becomes infeasible, i.e., before producing an entire abductive answer. Obtaining prediction/recommendation is slightly more complex. Consider, e.g., the request of an abductive answer that minimizes the overall execution time. A naive solution would compute all possible abductive answers, then choosing the one with minimum completion time. A branch-and-bound optimisation is obtained by adding the following rules, imposing that any observed/hypothesised event must always happen before the final event:

$$
\begin{aligned}
\mathbf{H}\left({ }_{-}, T_{1}\right) & \wedge \mathbf{H}\left(\text { out- } \mathrm{B}_{0}, T_{e}\right) \rightarrow T_{1}<T_{e} . \\
\mathbf{A B D}\left({ }_{-}, T_{1}\right) & \wedge \mathbf{H}\left(\text { out- } \mathrm{B}_{0}, T_{e}\right) \rightarrow T_{1}<T_{e} .
\end{aligned}
$$

Intuitively, as long as no solution is found, out- $\mathrm{B}_{0}$ is not hypothesised. As soon as a possible answer is found, it is. After that, all hypotheses are bound to happen before the best final time.

A prototype implementation of the framework is currently available for download at http://ai. unibo.it/AlpBPM

\section{Evaluation}

In this section we investigate the performance of each reasoning task separately by changing the input parameters of interest. The focus of our evaluation is to analyse the scalability of the proposed approach. The experiments have been carried out on a Windows 7 pc with 8GB RAM and a 2.4 GhZ Intel-core i7. The encoding presented in Section 3.1 has been adopted, but few optimizations have been applied to exploit some peculiar features of the SCIFF proof procedure implementation.

We encoded 5 process models $(M 1, \ldots, M 5)$ with different characteristics. $M 1$ is the model taken from [7] reported in Figure 1, while $M 2 \ldots M 5$ are synthetic models built from two or more replicas of $M 1$. For notation simplicity we denote with $K$ the core part of $M 1$, i.e., $M 1$ excluded the start and the end events, and we will enumerate the replicas of $K$ in $M 2 \ldots M 5$ with progressive numbers. In detail, $M 2$ concatenates two replicas of $K$, identified as $K 0$ and $K 1$ (see Figure 2(b)), $M 3$ composes two replicas $K 0$ and $K 1$ of $K$ in parallel (see Figure 2(c)), $M 4$ concatenates three copies of $K$ namely, $K 0, K 1$ and $K 2$, in sequence (see Figure 2(d) and, finally, $M 5$ consists of an exclusive choice between two copies $K 0$ and $K 1$ of $K$ in sequence and a copy $K 2$ of $K$ (see Figure 2(e) 8

\footnotetext{
${ }^{8}$ The expanded version of all the five models is available, together with their SCIFF encoding, at the link http://ai.
} 


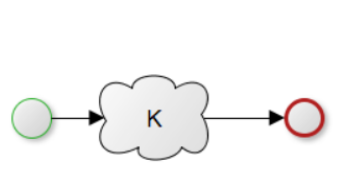

(a) $M 1$

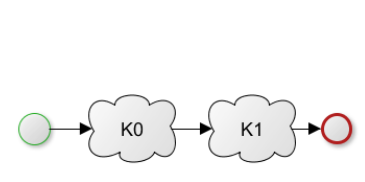

(b) $M 2$

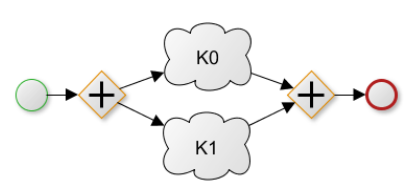

(c) $M 3$

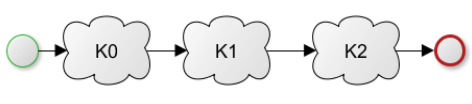

(d) $M 4$

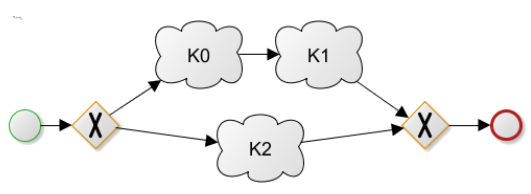

(e) $M 5$

Figure 2. Models $M 1, \ldots, M 5$ used in the evaluation

To disambiguate between the replicas of activities in the different models we will rename them specifying, for each original activity $A i$ belonging to the model in Figure 11, also the the $K j$ name of the replica of $K$ the activity belongs to. For instance, the first activity of $M 4$, which is also the first

\begin{tabular}{|c|c|c|c|c|c|c|c|}
\hline Model & Size & Paral. \% & Min/Max & \# TCs & Incons. Source & Out. & Time(s) \\
\hline \multirow{2}{*}{$M 1$} & \multirow{2}{*}{20} & \multirow{2}{*}{0.14} & \multirow{2}{*}{$6 / 11$} & \multirow{2}{*}{4} & & $c$ & 0.16 \\
\hline & & & & & $\operatorname{tcon}(\mathrm{A} 1, \mathfrak{s}, \mathrm{A} 2, \mathfrak{e})=\langle 0,5\rangle$ & $i$ & 0.17 \\
\hline \multirow{2}{*}{$M 2$} & \multirow{2}{*}{40} & \multirow{2}{*}{0.14} & \multirow{2}{*}{$12 / 22$} & \multirow{2}{*}{8} & & $c$ & 0.52 \\
\hline & & & & & $\operatorname{tcon}\left(\mathrm{A} 1_{\mathrm{K} 1}, \mathfrak{s}, \mathrm{A} 2_{\mathrm{K} 1}, \mathfrak{e}\right)=\langle 0,5\rangle$ & $i$ & 0.83 \\
\hline \multirow{2}{*}{ M3 } & \multirow{2}{*}{42} & \multirow{2}{*}{1} & \multirow{2}{*}{$12 / 22$} & \multirow{2}{*}{8} & & $c$ & 2.01 \\
\hline & & & & & $\operatorname{tcon}\left(\mathrm{A} 1_{\mathfrak{K} 1}, \mathfrak{s}, \mathrm{A} 2_{\mathrm{K} 1}, \mathfrak{e}\right)=\langle 0,5\rangle$ & $i$ & 0.66 \\
\hline \multirow{2}{*}{$M 4$} & \multirow{2}{*}{60} & \multirow{2}{*}{0.14} & \multirow{2}{*}{$18 / 33$} & \multirow{2}{*}{12} & & $c$ & 1.6 \\
\hline & & & & & $\operatorname{tcon}\left(\mathrm{A} 1_{\mathrm{K} 2}, \mathfrak{s}, \mathrm{A} 2_{\mathrm{K} 2}, \mathfrak{e}\right)=\langle 0,5\rangle$ & $i$ & 2.51 \\
\hline \multirow{3}{*}{$M 5$} & \multirow{3}{*}{62} & \multirow{3}{*}{0.14} & \multirow{3}{*}{$6 / 22$} & \multirow{3}{*}{12} & & $\mathrm{c}$ & 0.78 \\
\hline & & & & & $\operatorname{tcon}\left(\mathrm{A} 1_{\mathrm{K} 1}, \mathfrak{s}, \mathrm{A} 2_{\mathrm{K} 1}, \mathfrak{e}\right)=\langle 0,5\rangle$ & & 109 \\
\hline & & & & & $\operatorname{tcon}\left(\mathrm{A} 1_{\mathrm{K} 2}, \mathfrak{s}, \mathrm{A} 2_{\mathrm{K} 2}, \mathfrak{e}\right)=\langle 0,5\rangle$ & $\imath$ & 1.09 \\
\hline
\end{tabular}

Table 1. Model Consistency. activity of $K 0$, will be denoted as $A 1_{K 0}$; the first activity in $K 1$ will be indicated as $A 1_{K 1}$, while the first activity in $K 2$ will be named $A 1_{K 2}$. We encoded all tasks as partially observable, which is the worst case performance-wise, as we discuss later in the section.

Model consistency. Table 1 reports the characteristics of the models: size (column "Size"), i.e., the number of activities and gateways in BPMN, parallelism degree (column "Paral. \%"), i.e., the percentage of activities that can be executed in parallel, min/max path length in the model (column "Min/Max") and number of temporal constraints (column "\# TCs"). For each of these (consistent) models, an inconsistent variant is built by modifying one of the constraints. For each of the models, we modified the constraint between the first two activities of one of the replicas of $K$ (i.e., $\operatorname{tcon}\left(\mathrm{A} 1_{\mathrm{K} 0}, \mathfrak{s}, \mathrm{A} 2_{\mathrm{K} 0}, \mathfrak{e}\right)=\langle 0,30\rangle$ for $\left.K 0\right)$, by imposing that the time between the first and the second activity is instead between 0 and 5 time units (i.e., $\operatorname{tcon}\left(\mathrm{A} 1_{K 0}, \mathfrak{s}, \mathrm{A} 2_{\mathrm{K} 0}, \mathfrak{e}\right)=\langle 0,5\rangle$ for $\left.K 0\right)$, which generates an inconsistent model given the duration range of the first two activities. In detail, as reported in the column "Incons. Source" of Table 1 for $M 2$ and $M 3$ we changed the constraint related to $K 1$, for $M 4$ we changed the constraint in $K 2$ and for $M 5$ we changed the constraints both in $K 1$ and in $K 2$. In this way, we are able to test model inconsistencies occurring in different points of the 
model. Table 1 reports the outcome of the consistency check (column "Out") denoted with $c$, in case of consistency and $i$ in case of inconsistency, as well as the time (column "Time(s)") required by the abductive framework for assessing, respectively, the consistency or the inconsistency of $M 1 \ldots M 5$.

As expected, the results reported in the table show that the size and the structure of the model influence the time required for checking the (in)consistency. For instance, detecting the consistency of $M 3$, where a higher number of parallel gateways occurs, is more expensive than computing the consistency for models of larger size. Also, inconsistency detection requires overall more time than the consistency detection, as when no consistent path is found, all the paths of the model have to be explored. The only exception to this pattern occurs for the model with the parallel construct $M 3$. In this case, indeed, evaluating the interleavings while searching for a compliant solution could result being more expensive than identifying the inconsistency.

Strong compliance. For each model in $M 1 \ldots M 5$ we consider 2 compliant and 2 non compliant traces of different length. Table 2 reports, for each model (column "Model”), the 4 traces (column "Trace"), their length (column "Length") and the source of non-compliance (column "NC Source") for the non compliant traces, the outcome of the strong compliance procedure (column "Out."), i.e., $c$ for compliant or $n c$ for non-compliant, as well as the time required by the SCIFF procedure to compute the result (column "Time"). We notice that the time required for the strong compliance ranges between less than one second to less than one minute (about $50 \mathrm{sec}$.) for short/medium traces (e.g., up to 12 events), and increases up to more than one hour as the trace becomes longer. By looking at the results related to models with similar characteristics and increasing size, such as $M 1, M 2$ and $M 4$, we notice an exponential trend in computational time (see traces $\mathrm{t} 1 \_1$, $\mathrm{t} 2 \_1$ and $\left.\mathrm{t} 4 \_1\right)$. This is due to the fact that all tasks

\begin{tabular}{|c|c|c|c|c|c|}
\hline Model & Trace & Length & NC Source & Out & Time(s) \\
\hline \multirow{4}{*}{$M 1$} & $\mathrm{t} 1 \_1$ & 6 & & $c$ & 0.48 \\
\hline & $\mathrm{t} 1 \_2$ & 6 & $(\mathrm{~A} 4,[1,2])$ & $n c$ & 0.01 \\
\hline & $\mathrm{t} 1 \_3$ & 11 & & $c$ & 12.49 \\
\hline & $\mathrm{t} 1 \_4$ & 11 & $(\mathrm{~A} 6,[1,2])$ & $n c$ & 0.01 \\
\hline \multirow{4}{*}{$M 2$} & $\mathrm{t} 2 \_1$ & 12 & & $c$ & 357.08 \\
\hline & $\mathrm{t} 2 \_2$ & 12 & $\left(\mathrm{~A} 4_{\mathrm{K} 1},[1,2]\right)$ & $n c$ & 0.02 \\
\hline & $\mathrm{t} 2 \_3$ & 22 & & $c$ & $>1 \mathrm{~h}$ \\
\hline & $\mathrm{t} 2 \_4$ & 22 & $\left(\mathrm{~A} 13_{\mathrm{K} 0},[1,2]\right)$ & $n c$ & 0.032 \\
\hline \multirow{4}{*}{$M 3$} & t3_1 & 12 & & $c$ & 35.64 \\
\hline & $\mathrm{t} 3 \_2$ & 12 & $\left(\mathrm{~A}_{\mathrm{K} 1},[1,2]\right)$ & $n c$ & 0.05 \\
\hline & $\mathrm{t} 3 \_3$ & 22 & & $c$ & $>1 \mathrm{~h}$ \\
\hline & $\mathrm{t} 3 \_4$ & 22 & $\begin{array}{l}\left(\mathrm{A} 11_{\mathrm{K} 0},[159,250]\right) \\
\left(\mathrm{A} 11_{\mathrm{K} 1},[160,251]\right)\end{array}$ & $n c$ & 0.09 \\
\hline \multirow{4}{*}{$M 4$} & t4_1 & 18 & & $c$ & 2090.36 \\
\hline & $\mathrm{t} 4 \_2$ & 18 & $\left(\mathrm{~A}_{\mathrm{K}_{2}},[1,2]\right)$ & $n c$ & 0.02 \\
\hline & $\mathrm{t} 4 \_3$ & 33 & & $c$ & $>1 \mathrm{~h}$ \\
\hline & $\mathrm{t} 4 \_4$ & 33 & $\left(\mathrm{~A} 5_{K 2},[1,2]\right)$ & $n c$ & 0.06 \\
\hline \multirow{4}{*}{ M5 } & t5_1 & 6 & & $c$ & 0.76 \\
\hline & $\mathrm{t} 5 \_2$ & 6 & $\left(\mathrm{~A} 13_{\mathrm{K} 2},[3,4]\right)$ & $n c$ & 0.38 \\
\hline & $\mathrm{t} 5 \_3$ & 11 & & $c$ & 50.8 \\
\hline & t5_4 & 11 & $\left(\mathrm{~A} 10_{\mathrm{K} 2},[1,2]\right)$ & $n c$ & 0.06 \\
\hline
\end{tabular}

Table 2. Strong Compliance. in the models are partially observable, which requires "reasoning by cases" for each task in the model. Indeed, as explained in Section 3, since each event expectation can be matched with an abducible or with an actual observation in the trace, both options have to be considered when the event is partially observable. This cost can be avoided if the activity is marked as observable/unobservable.

Conditional compliance and runtime monitoring/prediction. We tested out, both conditional compliance and runtime monitoring with two levels of event incompleteness (50\% and $80 \%)$ on the 4 traces and the 5 models used for evaluating the strong compliance, for a total of 80 tests. While in the conditional compliance the missing events are distributed along the whole execution trace, in runtime monitoring and prediction incompleteness characterizes the last part of

\begin{tabular}{|cc|cc|ccc|}
\hline Model & Trace & Length & Inc. \% & Out. & $\begin{array}{c}\text { C. Compl. } \\
\text { Time(s) }\end{array}$ & $\begin{array}{c}\text { Monit. } \\
\text { Time(s) }\end{array}$ \\
\hline \multirow{4}{*}{$M 1$} & t1_1a & 6 & 50 & $c$ & 0.26 & 0.23 \\
& t1_1b & 6 & 80 & $c$ & 0.22 & 0.1 \\
& t1_2a & 6 & 50 & $n c$ & 0.01 & 0.21 \\
& t1_2b & 6 & 80 & $n c$ & 0.01 & 0.06 \\
& t1_3a & 11 & 50 & $c$ & 1.1 & 0.93 \\
& t1_3b & 11 & 80 & $c$ & 0.12 & 0.19 \\
& t1_4a & 11 & 50 & $n c$ & 0.01 & 0.61 \\
& t1 4b & 11 & 80 & $n c$ & 0.07 & 0.17 \\
\hline
\end{tabular}


the trace only (see the right-hand part of Figure 11. Table 3 summarizes the results of the two reasoning services: for each model and for each trace, the percentage of incompleteness of the considered traces is reported (column "Inc. $\%$ "). The incompleteness degree ranges between $50 \%$ (half of the events of a complete path from the source to the target is observed in the trace) and $80 \%$ (about $20 \%$ of the events of a path from the source to the target are observed in the trace). Notice that the case where all the events are observed (incompleteness degree $0 \%$ ) is covered by the strong compliance case. All compliant/non compliant traces used in this evaluation have indeed been obtained by removing activities from the ones used for the strong compliance check. Column "Out." reports the outcome of the service while columns "C. Compl" and "Monit. Time" the computation time for each of the two services. The computation times required for the two services are quite similar though with some differences for large models. Overall, the framework seems to be more efficient when the source of the incompleteness is located along the trace rather than at the end, in case of large models with a high degree of parallelism (e.g., see traces t3_3a and t3_4a for M3). Conversely, with large models characterized by a low parallelism degree, monitoring seems to overcome conditional compliance (e.g., see traces t4_3a and t4_4b for $M 4$ ). Although computing times are exponential in path length and model size as for the strong compliance, in this case we notice an improvement in performances compared to the strong compliance ones: the less events we observe in the trace the lower the computation time is. This happens because expectations of some events (the ones corresponding to missing events) immediately fail to be matched with happened events (as traces are incomplete). The procedure is hence forced to match these expectations with abducibles, ruling out options from reasoning by cases and improving the performances. Note that the time required for the prediction/recommendation service is the same of runtime monitoring.

Overall Observations. In this experimentation, we focused on the borderline case, in which no knowledge at all is available about the observability of the events (i.e., all the events are partially observable). Purpose of the experimentation was understanding the applicability of the approach in practical scenarios. Overall, in a realistic scenario as the temporal model $M 1$ presented in [7], all the reasoning services are carried out in less than 15 seconds. When no information is known about 


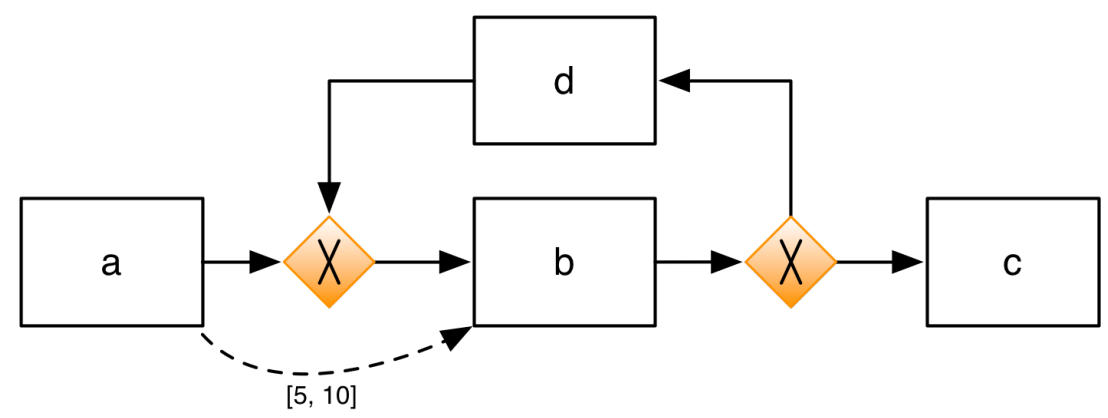

Figure 3. Loops and Temporal Constraints.

the observability of the events, the abductive framework is particularly performant in situations in which the execution trace is heavily incomplete either along or at the end of the trace. When a large part of the execution trace is unknown (e.g., about $80 \%$ of the trace), indeed, even for long paths the performances of the abductive framework are of the order of minutes.

\section{Extending SPOTs with Loops}

As pointed out in Section 2.1, well-defined SPOT models (as for Definition 2.2) do not allow the specification of loops in the workflow. The main reason for focusing on a workflow model preventing the repetition of tasks is the lack of an established semantics for temporal constraints between two activities that are part of a loop. For example, the workflow modelling language proposed in [7] (used in Figure 11 provides a rich formalism for expressing temporal constraints related to the duration of single activities, as well as inter-task temporal constraints, but no semantics for loops and temporal constraints is given. Another example is the language used in [16] for medical temporal workflows where, again, no semantics is proposed for temporal constraints involving activities in loops.

To understand the semantic issues that could arise in the case that a task can be repeated several times let us consider the workflow excerpt in Figure 3, where an inter-task constraint has been added between the activity a (not belonging to the loop path) and the activity $b$ (belonging to the loop path). The semantics of the inter-task constraint is ambiguous, since task $b$ can appear more than once in a trace. The constraint could refer to the first appearance of $b$, or to the last. At least three possible alternatives choices can be considered:

1. the constraint refers to all the occurrences of activity $b$ that happen after the execution of $a$;

2. the constraint refers to the first occurrence of $b$;

3. the constraint refers to the last occurrence of b.

Independently that the activities are observable or not, all the alternatives seem plausible: possibly, the "best choice" could be made only with sufficient insight on the specific application domain. Finally, more complex cases might arise: inter-task constraints could be about, for example, tasks that are both involved in two (distinct) loops: in such a case, similar considerations would be extended to both the activities. Note that the above ambiguity arises also in the case that we prevent constraints to cross the 


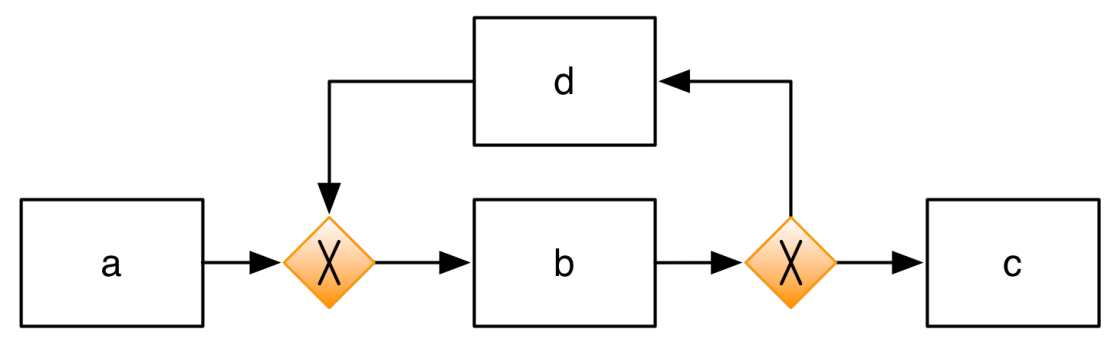

Figure 4. An excerpt of a simple workflow including a loop.

boundaries of a loop block; although it could be argued that in this case applying the constraint to the closest pair of occurring tasks might be a sensible choice.

The discussion and adoption of a specific semantics for temporal constraints in the presence of task repetitions is outside the scope of this work; however our approach can be extended in order to accomodate a looping construct. In the previous work [23] we discussed how to model workflow loops (translation details can be found in a technical report [24]). Here we briefly outline the main idea behind the encoding of loops. Let us consider a workflow excerpt shown in Figure 4 . Upon the end of activity b, either $c$ or $d$ is expected to be executed, with the workflow path containing $d$ being a loop path. Moreover, let us suppose also that all the activities are observable. The exclusive split block, relating tasks b, c, and d, can be modelled in SCIFF as follows (for the sake of comprehension, we omit here the rules concerning the in/out of blocks):

$$
\begin{gathered}
\mathbf{H}\left(\operatorname{end}(\mathrm{b}), T_{b}\right) \rightarrow \mathbf{E}\left(\operatorname{start}(\mathrm{c}), T_{c}\right) \wedge T_{c} \geq T_{b} \vee \mathbf{E}\left(\operatorname{start}(\mathrm{d}), T_{d}\right) \wedge T_{d} \geq T_{b} \\
\mathbf{H}\left(\operatorname{start}(\mathrm{c}), T_{c}\right) \wedge \mathbf{H}\left(\operatorname{start}(\mathrm{d}), T_{d}\right) \wedge T_{d}>T_{c} \rightarrow \perp
\end{gathered}
$$

The constraint (59) ensures that, upon the termination of task b, one task among c and d is executed. Constraint 60 instead guarantees that once the control flow has exited the loop, it is not possible to execute d any more. Constraints (10) and (11), introduced in Section 3.1, should be removed: they were introduced to ensure that, for each activity, only one execution is allowed in the trace. However, as a consequence of loops, certain activities will appear more than once. Moreover, we need to add two further constraints: for every activity, between two starts of the same activity, we should observe a completion of the same activity. Hence, given a generic task a, we would write:

$$
\begin{aligned}
& \mathbf{H}\left(\operatorname{start}(\mathrm{a}), T_{1}\right) \wedge \mathbf{H}\left(\operatorname{start}(\mathrm{a}), T_{2}\right) \wedge T_{2}>T_{1} \rightarrow \mathbf{E}\left(\operatorname{end}(\mathrm{a}), T_{e}\right) \wedge T_{e}>T_{1} \wedge T_{e}<T_{2} \\
& \mathbf{H}\left(\operatorname{end}(\mathrm{a}), T_{1}\right) \wedge \mathbf{H}\left(\operatorname{end}(\mathrm{a}), T_{2}\right) \wedge T_{2}>T_{1} \rightarrow \mathbf{E}\left(\operatorname{start}(\mathrm{a}), T_{s}\right) \wedge T_{s}>T_{1} \wedge T_{s}<T_{2}
\end{aligned}
$$

Notice that in case we would allow the nesting of loop blocks, the formalisation would become more complex, due to the fact that we could observe multiple executions of the same loop block. For example, a unique identifier might be needed to distinguish between multiple block execution instances.

In the case the activities are non-observable, or partially observable, further constraints would be added in a similar way to constraints (59)-(62). However, termination issues might arise when abducing events (in the loop path) that have not been observed: to avoid to abduce an infinte number 
of events, SCIFF has been implemented to explore the non-loop path as a first hypothesis for conformance, and to explore the loop path only when the former is detected to be not compliant. Moreover, we require that either the maximum length of a trace is given as a input parameter, or that the timestamp of the end activity is known: in both cases, SCIFF algorithm will terminate.

\section{Related Work}

A consistent part of the BPM literature of the last decades has been devoted to provide reasoning services, such as process model consistency, trace compliance, runtime monitoring as well as scheduling/planning, on top of process models and their executions. Consistency of process models is investigated in a number of works, and particularly in the case of workflows enriched with different types of constraints, as for instance business contracts [5] or time [25, 26] constraints. For example, Bettini et al. [25] suggest an approach based on Simple Temporal Networks (STN), where each node represents a time point, edges are temporal relationships and each task is represented by a start and an end node. Planning and critical path methods are exploited instead by Eder and colleagues [26] where the compliance of workflow models is checked in presence of conditional activities.

The (strong) compliance of traces towards process models (also known as conformance checking) is another key reasoning service, as suggested by the several approaches proposed for dealing with the disalignment between models and traces looking at the control flow only [27, 28] or taking into account also data [29] or time constraints [30]. For example, in [30], compliance to time-constrained workflows is verified with a two-step procedure: the trace is first aligned to the model and then to the time constraint rules. A different perspective on the (strong) compliance is offered in [31], where potential temporal anomalies are detected with respect to a Bayesian model (automatically inferred from a Petri Net) enriched with temporal annotations extracted from historical execution traces.

Conditional compliance of traces towards process models has been tackled in the broader field of conformance checking, although in an indirect manner. Indeed, a number of works focus on the alignment of event logs and procedural/declarative process models: for example, [28, 32] explore the search space of the set of possible moves to find the best ones for aligning the log to the model. Conditional compliance is then viewed as a problem of alignment. Rather, the notion of conditional compliance adopted in this work focuses on prescriptive models and on incomplete logs. Only few approaches in the BPM literature more closely relate to such a notion, for example by leveraging on techniques as Satisfiability Modulo Theory [33], or planning techniques [34].

A number of approaches and tools have been proposed for the runtime monitoring, and applied for checking the compliance of running traces versus (enriched) process models, taking into consideration control-flow aspects [4], control-flow and data [35], control-flow and time [16]. For example, Combi and colleagues [16] propose a conceptual model for temporal processes, provide different types of design-time and runtime compliance, and introduce ad-hoc techniques for checking these different levels of compliance. Concerning prediction/recommendation services, some effort has been devoted to recommend how to optimize dimensions such as resources [36] or time [37, 7]. In particular, the work in [7] (from which the example in Figure 1 is taken) presents an approach based on control-flow and temporal constraint satisfaction for the runtime monitoring as well as for the identification of the schedule for a case that better minimizes the time constraint violations. 
The SCIFF framework, and in particular its support to abduction, have been exploited in the past to model both procedural [38] and declarative [39] processes, without considering the issue of incompleteness. In [23] instead we focused on the incompleteness issue only, discussing the different types of incomplete data that can be observed in real logs. However, in that work we did not discuss the temporal dimension, that was instead addressed for the first time in a poster presented at ECAI [1]. Hence, [23, 1] can be considered as initial steps towards the SPOT model introduced here. Interestingly, in [40] abduction is exploited to evaluate trace compliance, but checking user permission compliance only. Moreover, [40] deals with incomplete traces only (with complete events), while our solution takes a more sophisticated approach to incompleteness and reasoning services. Note also that the adopted abductive framework, CIFF [41], only supports ground abducibles and denial constraints.

The majority of works on temporal processes assume that the environment is fully observable and that the duration of each activity (as well as the other temporal constraints) are certain and under control of the system. Real world situations, however, show that activity durations are not always controllable, thus motivating the need of dealing with uncertainty in dynamic environments. A lot of research has been devoted to these issues: for example, in [42] the definition of Simple Temporal Networks with Uncertainty is given as an extension of Simple Temporal Networks [43], with distinction and support to controllable and uncontrollable events. Recent works deal with temporal planning aspects with uncertainty [44], and with the scheduling of multi-task applications [45] with uncertainty as well. Currently, our approach deals only with the partial observability of the environment, and the controllability issue is not taken into account.

Within the Artificial Intelligence field a number of alternative approaches to incompleteness exist, as well as other frameworks that support abductive reasoning (e.g., ASP [46]), which we will consider in future work. The aim of this contribution is to show that abduction, and in particular Abductive Logic Programming, is a natural choice for representing the problem of observed, incomplete traces. In addition, key features of SCIFF, such as native support of notions like expectations and fulfilment/violation of traces against model's prescriptions, make the formalism especially appealing to reason with the problem at hand.

\section{Conclusions}

We have presented an abductive framework to support business process compliance, by attacking the different forms of incompleteness that may be present in an execution trace, and by supporting also the temporal dimension in terms of constraints on the activity durations and between different activities. To this purpose, we introduced the notion of SPOT model, a coherent encoding into the SCIFF framework, and showed how different reasoning tasks can be addressed through the SCIFF proof procedure. A current limit is that SPOT models do not support loops. This is due to the lack of a clear semantics for temporal constraints between two activities: particularly ambiguous is the case when such a constraint connects an activity involved in a loop. In previous work [23] we showed how to deal with loops using an encoding similar to the one presented here: however, [23] ignored the temporal dimension.

Concerning future development, the SCIFF framework is based on first-order logic, thus paving the way towards the incorporation of data [35] and the management of more sophisticated forms of 
incompleteness. A further reasoning service on temporal workflows is the one of controllability. We believe that an extension of our work to deal with dynamic controllability, for instance integrating constraint propagation and filtering as in [45], would be an interesting and feasible future work.

\section{Acknowledgments}

This research has been partially supported by the Euregio IPN12 “KAOS: Knowledge-Aware Operational Support" project, which is funded by the "European Region Tyrol-South Tyrol-Trentino" (EGTC) under the first call for basic research projects. Marco Montali has been partially supported by the UNIBZ project KENDO: Knowledge-driven ENterprise Distributed cOmputing. Sergio Tessaris has been partially supported by the UNIBZ project PWORM: Planning for Workflow Management.

\section{References}

[1] Chesani F, De Masellis R, Francescomarino CD, Ghidini C, Mello P, Montali M, Tessaris S. Abducing Workflow Traces: A General Framework to Manage Incompleteness in Business Processes. In: Kaminka GA, Fox M, Bouquet P, Hüllermeier E, Dignum V, Dignum F, van Harmelen F (eds.), ECAI 2016 - 22nd European Conference on Artificial Intelligence, 29 August-2 September 2016, The Hague, The Netherlands - Including Prestigious Applications of Artificial Intelligence (PAIS 2016), volume 285 of Frontiers in Artificial Intelligence and Applications. IOS Press. ISBN 978-1-61499-671-2, 2016 pp. 1734-1735. doi:10.3233/978-1-61499-672-9-1734. URL http://dx.doi.org/10.3233/ 978-1-61499-672-9-1734.

[2] van der Aalst WMP, et al. Process Mining Manifesto. In: BPM Workshops. Springer, 2012 .

[3] van der Aalst WMP. Business Process Management: A Comprehensive Survey. ISRN Software Engineering, 2013. doi:10.1155/2013/507984. Vol. 2013, Article ID 507984, 37 pages, 2013. doi: $10.1155 / 2013 / 507984$.

[4] Maggi FM, Montali M, van der Aalst WMP. An Operational Decision Support Framework for Monitoring Business Constraints. In: FASE, volume 7212 of LNCS. Springer, 2012 pp. 146-162.

[5] Governatori G, Milosevic Z, Sadiq S. Compliance Checking Between Business Processes and Business Contracts. In: Proc. of EDOC. IEEE Computing Society, 2006 pp. 221-232.

[6] Combi C, Oliboni B, Zerbato F. Modeling and handling duration constraints in BPMN 2.0. In: Seffah A, Penzenstadler B, Alves C, Peng X (eds.), Proceedings of the Symposium on Applied Computing, SAC 2017, Marrakech, Morocco, April 3-7, 2017. ACM. ISBN 978-1-4503-4486-9, 2017 pp. 727-734. doi: 10.1145/3019612.3019618. URL http://doi.acm.org/10.1145/3019612.3019618

[7] Kumar A, Sabbella SR, Barton RR. Business Process Management: 13th International Conference, BPM 2015, Innsbruck, Austria, August 31 - September 3, 2015, Proceedings, chapter Managing Controlled Violation of Temporal Process Constraints, pp. 280-296. Springer International Publishing, Cham. ISBN 978-3-319-23063-4, 2015. doi:10.1007/978-3-319-23063-4\_20. URL http://dx.doi.org/10.1007/ 978-3-319-23063-4_20.

[8] Zavatteri M, Combi C, Posenato R, Viganò L. Weak, Strong and Dynamic Controllability of AccessControlled Workflows Under Conditional Uncertainty. In: Carmona J, Engels G, Kumar A (eds.), Business Process Management - 15th International Conference, BPM 2017, Barcelona, Spain, September 10-15, 
2017, Proceedings, volume 10445 of Lecture Notes in Computer Science. Springer. ISBN 978-3-31964999-3, 2017 pp. 235-251. doi:10.1007/978-3-319-65000-5_14. URL https://doi.org/10.1007/ 978-3-319-65000-5_14

[9] Rogge-Solti A, Mans R, van der Aalst W, Weske M. Improving Documentation by Repairing Event Logs. In: The Practice of Enterprise Modeling, volume 165 of LNBIP, pp. 129-144. Springer. ISBN 978-3-642-41640-8, 2013. doi:10.1007/978-3-642-41641-5_10. URL http://dx.doi.org/10.1007/ 978-3-642-41641-5_10

[10] Di Francescomarino C, Ghidini C, Tessaris S, Sandoval IV. Completing Workflow Traces using Action Languages. In: Proceedings of the 27th International Conference on Advanced Information Systems Engineering (CAiSE 2015), volume 9097 of Lecture Notes in Computer Science. Springer, 2015 pp. 314 330.

[11] Mannhardt F, de Leoni M, Reijers HA. Extending Process Logs with Events from Supplementary Sources, pp. 235-247. Springer International Publishing, Cham. ISBN 978-3-319-15895-2, 2015. doi:10.1007/ 978-3-319-15895-2_21. URL https://doi.org/10.1007/978-3-319-15895-2_21.

[12] Kakas AC, Kowalski RA, Toni F. Abductive Logic Programming. J. Log. Comput., 1992. 2(6).

[13] Denecker M, Kakas AC. Abduction in Logic Programming. In: Kakas AC, Sadri F (eds.), Computational Logic: Logic Programming and Beyond, Essays in Honour of Robert A. Kowalski, Part I, volume 2407 of Lecture Notes in Computer Science. Springer. ISBN 3-540-43959-5, 2002 pp. 402-436. doi:10.1007/ 3-540-45628-7\_16.

[14] Alberti M, Chesani F, Gavanelli M, Lamma E, Mello P, Torroni P. Verifiable agent interaction in abductive logic programming: The SCIFF framework. ACM Trans. Comput. Log., 2008. 9(4).

[15] Kiepuszewski B, ter Hofstede AHM, Bussler CJ. On Structured Workflow Modelling. In: Seminal Contributions to Information Systems Engineering. Springer, 2013.

[16] Combi C, Gozzi M, Posenato R, Pozzi G. Conceptual modeling of flexible temporal workflows. TAAS, 2012. 7(2):19.

[17] Kakas AC, Mancarella P. Abduction and Abductive Logic Programming. In: Proc. of ICLP. 1994.

[18] Fung TH, Kowalski RA. The Iff Proof Procedure for Abductive Logic Programming. J. Log. Program., 1997. 33(2).

[19] Kunen K. Negation in Logic Programming. J. Log. Program., 1987. 4(4).

[20] Clark KL. Negation as Failure. In: Logic and Data Bases. Plenum Press, 1978.

[21] Jaffar J, Maher MJ, Marriott K, Stuckey PJ. The Semantics of Constraint Logic Programs. J. Log. Program., 1998. 37(1-3).

[22] Alberti M, Chesani F, Gavanelli M, Lamma E, Mello P, Torroni P. Verifiable Agent Interaction in Abductive Logic Programming: the SCIFF proof-procedure. Technical Report DEIS-LIA-06-001, University of Bologna (Italy), 2006. LIA Series no. 75.

[23] Chesani F, De Masellis R, Francescomarino CD, Ghidini C, Mello P, Montali M, Tessaris S. Abducing Compliance of Incomplete Event Logs. In: Adorni G, Cagnoni S, Gori M, Maratea M (eds.), AI*IA 2016: Advances in Artificial Intelligence - XVth International Conference of the Italian Association for Artificial Intelligence, Genova, Italy, November 29 - December 1, 2016, Proceedings, volume 10037 of Lecture Notes in Computer Science. Springer. ISBN 978-3-319-49129-5, 2016 pp. 208-222. doi: 10.1007/978-3-319-49130-1_16. URL http://dx.doi.org/10.1007/978-3-319-49130-1_16. 
[24] Chesani F, De Masellis R, Di Francescomarino C, Ghidini C, Mello P, Montali M, Tessaris S. Abducing Compliance of Incomplete Event Logs. Technical Report submit/1584687, arXiv, 2016.

[25] Bettini C, Wang XS, Jajodia S. Temporal Reasoning in Workflow Systems. Distributed and Parallel Databases, 2002. 11(3):269-306.

[26] Eder J, Gruber W, Panagos E. Temporal modeling of workflows with conditional execution paths. In: Database and Expert Systems Applications. Springer, 2000 pp. 243-253.

[27] Rozinat A, van der Aalst WMP. Conformance Checking of Processes Based on Monitoring Real Behavior. Inf. Syst., 2008. 33(1):64-95.

[28] Adriansyah A, van Dongen BF, van der Aalst WMP. Conformance Checking Using Cost-Based Fitness Analysis. In: Proc. of EDOC. IEEE Computer Society, 2011.

[29] de Leoni M, van der Aalst W, van Dongen BF. Data- and Resource-Aware Conformance Checking of Business Processes. In: Proc. of BIS. Springer, 2012. doi:10.1007/978-3-642-30359-3\_5.

[30] Taghiabadi ER, Fahland D, van Dongen BF, van der Aalst WMP. Diagnostic Information for Compliance Checking of Temporal Compliance Requirements. In: CAiSE, volume 7908 of LNCS. Springer. ISBN 978-3-642-38708-1, 2013 pp. 304-320.

[31] Rogge-Solti A, Kasneci G. Temporal Anomaly Detection in Business Processes. In: Business Process Management - 12th International Conference, BPM 2014, Haifa, Israel, September 7-11, 2014. Proceedings. 2014 pp. 234-249. doi:10.1007/978-3-319-10172-9\_15.

[32] De Leoni M, Maggi FM, van der Aalst WMP. Aligning event logs and declarative process models for conformance checking. In: Proc. of BPM. Springer, 2012.

[33] Bertoli P, Di Francescomarino C, Dragoni M, Ghidini C. Reasoning-Based Techniques for Dealing with Incomplete Business Process Execution Traces. In: Proc. of AI*IA. Springer, 2013.

[34] Di Francescomarino C, Ghidini C, Tessaris S, Sandoval IV. Completing Workflow Traces Using Action Languages. In: Proc. of CAiSE. Springer, 2015.

[35] De Masellis R, Maggi FM, Montali M. Monitoring data-aware business constraints with finite state automata. In: Proc. of ICSSP. ACM Press, 2014 doi:10.1145/2600821.2600835.

[36] Barba I, Weber B, Del Valle C. Supporting the Optimized Execution of Business Processes through Recommendations. In: Business Process Management Workshops (1), volume 99 of LNBIP. Springer, 2011 pp. 135-140.

[37] van der Aalst WMP, Schonenberg MH, Song M. Time Prediction Based on Process Mining. Inf. Syst., 2011. 36(2):450-475. doi:10.1016/j.is.2010.09.001.

[38] Chesani F, Mello P, Montali M, Storari S. Testing Careflow Process Execution Conformance by Translating a Graphical Language to Computational Logic. In: Proc. of AIME. 2007 .

[39] Montali M, Pesic M, van der Aalst WMP, Chesani F, Mello P, Storari S. Declarative specification and verification of service choreographiess. TWEB, 2010. 4(1).

[40] Mian US, den Hartog J, S Etalle NZ. Auditing with incomplete logs. In: Proc. of HotSpot. 2015.

[41] Mancarella P, Terreni G, Sadri F, Toni F, Endriss U. The CIFF proof procedure for abductive logic programming with constraints: Theory, implementation and experiments. TPLP, 2009. 9(6).

[42] Vidal T, Fargier H. Handling contingency in temporal constraint networks: from consistency to controllabilities. Journal of Experimental and Theoretical Artificial Intelligence, 1999. 11:23-45. 
[43] Dechter R, Meiri I, Pearl J. Temporal constraint networks. Artificial Intelligence, 1991. 49(1):61-95.

[44] Cimatti A, Micheli A, Roveri M. Strong Temporal Planning with Uncontrollable Durations: A State-Space Approach. In: Proc.of AAAI. 2015 pp. 3254-3260.

[45] Lombardi M, Milano M, Benini L. Robust Scheduling of Task Graphs under Execution Time Uncertainty. IEEE Trans. Computers, 2013. 62(1):98-111.

[46] Baral C. Knowledge Representation, Reasoning, and Declarative Problem Solving. Cambridge University Press, New York, NY, USA, 2003. ISBN 0521818028. 